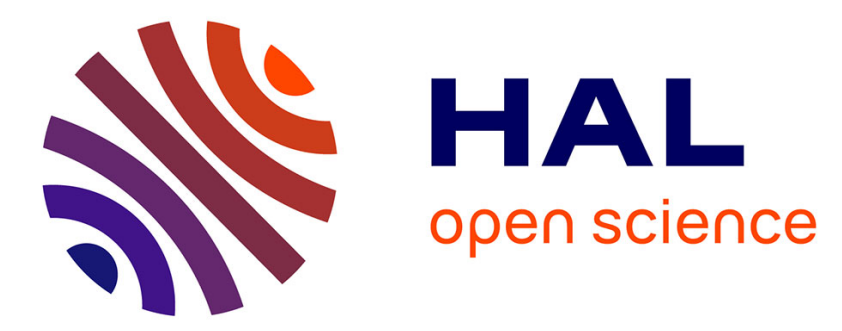

\title{
A multi-scale approach to investigate the non linear subsurface behavior and strain localization of X38CrMoV5-1 martensitic tool steel: experiment and numerical analysis
}

Ahmed Zouaghi, Vincent Velay, Adriana Soveja, Thomas Pottier, Mohammed Cheikh, Farhad Rezai-Aria

\section{To cite this version:}

Ahmed Zouaghi, Vincent Velay, Adriana Soveja, Thomas Pottier, Mohammed Cheikh, et al.. A multiscale approach to investigate the non linear subsurface behavior and strain localization of X38CrMoV51 martensitic tool steel: experiment and numerical analysis. International Journal of Plasticity, 2016, 87, pp.130-153. 10.1016/j.ijplas.2016.09.007 . hal-01398857

\section{HAL Id: hal-01398857 https://hal.science/hal-01398857}

Submitted on 17 Nov 2016

HAL is a multi-disciplinary open access archive for the deposit and dissemination of scientific research documents, whether they are published or not. The documents may come from teaching and research institutions in France or abroad, or from public or private research centers.
L'archive ouverte pluridisciplinaire HAL, est destinée au dépôt et à la diffusion de documents scientifiques de niveau recherche, publiés ou non, émanant des établissements d'enseignement et de recherche français ou étrangers, des laboratoires publics ou privés. 


\title{
A multi-scale approach to investigate the non linear subsurface behavior and strain localization of X38CrMoV5-1 martensitic tool steel: experiment and numerical analysis
}

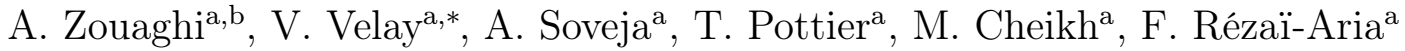 \\ ${ }^{a}$ Université de Toulouse; CNRS, Mines Albi, INSA, UPS, ISAE, ICA (Institut Clément Ader), \\ Campus Jarlard, 81013 Albi Cedex 09, France \\ ${ }^{b}$ ArcelorMittal, Global R\&D Maizières Research, BP 30320, 57283 Maizières-lès-Metz Cedex, \\ France
}

\begin{abstract}
The cyclic mechanical behavior, the wear and fatigue resistances and damage developments of working surface of tool steels are dependent on microstructural features. A multi-scale approach combining experimental testing, numerical treatments and simulations is developed to model the surface behavior of X38CrMoV5-1 martensitic tool steels. The multi-scale modeling is coupled with finite element calculations. The elasto-viscoplastic constitutive equations used are based on crystal plasticity model of Méric-Cailletaud and are implemented on the finite element code ABAQUS under a small strain assumption. Trough an appropriate laboratory testing, the microstructure features comparable to the surface of industrial tools or pin/disc in tribology experiments are reproduced by considering plate specimens. Monotonic tensile testing is coupled with in-situ Digital Image Correlation technique (DIC) to determine the surface strain fields. The measured local nonlinear mechanical strain fields are analyzed. The strain localization is related to stereological artifacts. The numerical treatments allow reproducing, qualitatively, the strain localization patterns at the surface observed during tensile testing. The influence of the various stereological parameters such as the morphology of martensitic laths, the crystallographic orientations, the internal hardening state of the surface profiles and their evolutions on the local strain fields are addressed. By such approach, it is possible to get a better insight of some elementary mechanisms acting on tools and/or pin/disc surfaces regarding both tensile and cyclic behavior.
\end{abstract}

Keywords: A.microstructures, B.crystal plasticity, B.cyclic loading, C.mechanical testing, surface behavior

\section{1. Introduction}

2 During forming operations such as forging, rolling, stamping ..., and more specif3 ically at high temperatures, the tool surfaces experience thermal and mechanical

\footnotetext{
*Corresponding author

Email address: vincent.velay@mines-albi.fr (V. Velay)
} 
cyclic loadings under transient conditions. Fatigue and wear are the two main damage mechanisms of tool surfaces. Depending on tool geometry and local thermomechanical loading, fatigue or wear may become the leading damage mechanism. At the stress raiser regions (e.g. tool radii, holes, ...) uni-axial cracking is generally dominant while the local loading is at least bi-axial state on plane surface and the interconnected cracks pattern called heat checking, is mostly observed in these regions. The tool surface is subjected to local plastic yielding especially at singularity locations (e.g. corners and stress raisers). These damage mechanisms can also act simultaneously and in coupling with environment such as oxidation or corrosion (in die casting). The prediction of the tool life is of primary concern for tool designing and damage monitoring. The interruptions in production because of premature tools damaging are very time consuming in term of reparation and are highly cost effective. Therefore, the lifetime prediction is a major issue for optimizing the tool design. These approaches require relevant thermo-mechanical constitutive models. However, such models are generally addressed using a RVE (Representative Volume Element) features and consider the material as isotropic in behavior. Nevertheless, almost all tool surfaces present very early microstructure texturing at different scales : macroscopic, mesoscopic and microscopic. Such microstructural evidences show that constitutive laws must take into account the anisotropic behavior near the working surfaces. It should be emphasized that such changes in extreme surface of tools are very common features that cover many surfaces of bodies in relative movements and presenting certain shear ductility. In fact, in contact surface, a few grains bear the whole load. As the elasto-plastic behavior of a grain is physically dominated by the nature of the crystal lattice and its actual orientation regarding the local main loading axis, it is senseless to consider an isotropic constitutive law. By definition the surfaces are singular for chemical reactions, mechanical behaviors and damage developments. Therefore, a special attention must be paid to the anisotropic nature of the surface when it experiences shearing. Thus, crystal plasticity models with multi-scale approaches have to be addressed. They can improve the description of the local behavior at tool surface vicinity $(\approx 100 \mu \mathrm{m})$, while classical macroscopic approaches are worthless for such matter. They include a local behavior model able to take into account both representative external loading and the slip system interactions of the martensitic BCC (Body-Centered Cubic) microstructure [1] as is the case for hot work tool steels. Some elementary physical mechanisms are introduced in these approaches for describing the cyclic plasticity [2 4] through a non linear kinematic variable [5, 6]. These models can be improved by taking into account the influence of the total dislocation densities with strain for each slip system [7] and by introducing an isotropic hardening variable [8, 9]. Different yield surfaces can be associated to the physical phenomena like the screw and edge dislocation effects [10,11]. However, the parameters identification of such models is not trivial. Phenomenological approaches are based on thermodynamics of the irreversible process. The constitutive equations are very similar to the formulations addressed in the physical approaches. Again, the shear strain rate is related to the resolved shear stress [12]. Many investigations consider an isotropic hardening variable to describe different slip systems interaction by introducing latent and self hardening mechanisms [13 -16]. The kinematic hardening can be included by 
using the Armstrong-Frederick or Chaboche equations [17]. The model parameters can then be identified using either a mean field or a full field approach. Berveiller and Zaoui [18] propose a scale tansition rule to describe the monotonic behavior of spherical particules. Later, Pilvin and Cailletaud propose an extension of the Berveiller-Zaoui model, the $\beta$-model [19], that accounts for cyclic loadings. The mean field approaches are relevant for identifying the material parameters at a local scale. However, they strongly depend on the linearisation methods used at the local and global scales. For this purpose, full fields approaches are more accurate and better describe the behavior of a given RVE. They explicitly take into account the internal structure of the material (morphology, crystallography, nature ...) [3, 4] and provide a spatial localization of mechanical fields [20 26]. Obviously, the resulting calculations are more reliable than those induced by a mean field model though being more expensive. One of the method employed in scale transition rule is FE (Finite Element) method. It consists in solving a macroscopic boundary problem in coupling with RVE which considers the actual microstructure features of the material. In such approaches, the constitutive equations are solved at each integration point of the FE mesh. The strain fields obtained at a local scale can be compared to local experimental measurements by Digital Image Correlation techniques [27 31]. In this frame, the $\mathrm{FE}$ analysis can be used to assess the reliability of the mean field simulations [32].

In the present investigation, the Meric and Cailletaud model [33 35] is used for predicting the surface behavior of hot forming tools (e.g. forging) and pin-on-disc tests [36, 37]. It should be emphasized that the surface of hot forming tools experiences actually transient thermo-mechanical wear and fatigue solicitations. The crystal plasticity models are adequate to take into account the crystallography features such as plastic anisotropy [38] and temperature dependence of physical and mechanical properties in particular for BBC and tetragonal lattice structures like martensitic material reported here [39]. Due to experimental difficulties for high temperature strain field measurements, the model capability was first assessed at room temperature. Nevertheless, the model is expressed for being easily applied both at isothermal and transient thermomechanical conditions. The mean field approach is performed for parameter identification sake. The scale transition rules, Berveiller-Zaoui and $\beta$ models are considered. The results are compared with those obtained by a full field approach. This latter uses a FE calculation at the scale of the RVE. In this case, the actual microstructure of tool steel is explicitly taken into account. The constitutive equations are implemented on ABAQUS software. The martensitic microstructure is modeled by using Voronoï tesselation and Neper software [40]. A parametric analysis is undertaken to assess the effect of several factors, namely: surface hardening, surface anisotropy, crystallographic orientation, grain/lath morphology, on the local and global induced mechanical fields. All these criteria can influence the surface properties and the material [4]. In some cases, a geometrically realistic microstructure can have more important effects on the heterogeneous deformation processes than a fine tuning of the constitutive model parameters [41]. A recent investigation have shown that a multiscale framework with an explicit representation of tempered martensitic microstructure accurately describes the typical softening effects due to precipitate and lath coarsening [42]. In this study, 
the local behavior model allows to take into account the laths shape and the slip systems interactions of the BCC martensitic microstructure. Two kinds of experiments are conducted. First, tests are performed on flat specimens to generate the typical surface observed on industrial tools. These specimens experience monotonic tensile loading. The surface roughening and its influence on the strain heterogeneity and grain localization is investigated since these interactions can have a significant effect on the mechanical behavior [43]. Multiscale modeling is a relevant approach to catch such kind of phenomena [44]. In this frame, DIC technique allows high resolution strain mapping [45] and therefore are relevant to compare the local measured strain fields with the results provided by the FE simulation. The interplay between simulation results and experiment provided by full field measurements at a local scale is not trivial to perform especially when complex microstructures are considered [46]. Last, the multi-scale modeling is extended to describe the cyclic behavior and the results are analyzed regarding the previous factors investigated under monotonic conditions.

\section{Experimental procedures}

\subsection{Material}

The material investigated is a $5 \%$ chromium double tempered martensitic steel (X38CrMoV5-1, AISI H11). Its chemical composition is reported in table 1.

Table 1: Chemical composition wt.\%

\begin{tabular}{cccccccc}
\hline $\mathrm{C}$ & $\mathrm{Cr}$ & $\mathrm{Mn}$ & $\mathrm{V}$ & $\mathrm{Ni}$ & $\mathrm{Mo}$ & $\mathrm{Si}$ & $\mathrm{Fe}$ \\
\hline 0.4 & 5.05 & 0.49 & 0.47 & 0.2 & 1.25 & 0.92 & Bal. \\
\hline
\end{tabular}

The heat treatments results in an initial hardness of $47 \mathrm{HRC}$ with a tempered martensitic lath microstructure. In fact, the microstructure consists of ferrite and cementite in form of lath (Fig. 1). The lath morphology is quite heterogeneous with an average thickness less than $2 \mu \mathrm{m}$. The laths are arranged by packets within the Prior Austenitic Grains (PAG) whose mean size is around $30 \mu \mathrm{m}$. EBSD (Electron BackScatter Diffraction) analysis have revealed that global crystallographic orientations of the grain is isotropic which confer to the steel an isotropic behavior. However, following early motion between tool and formed material (alt. between pin and disc), the grains are stretched along the sliding direction. By EBSD, the crystallographic orientation relationships of the martensitic laths (Body-Centered Cubic) and the PAG (Face-Centered Cubic) lattices are identified. Several approaches can be found to assess these orientation relationships [47, 48]. In the present study, the PAG wherein the martensitic lath blocks are stacked, is analyzed by using the crystallographic orientations. It is observed that the morphology of the martensitic laths are arranged in a quasi-parallel manner (see Fig. 2). The microstructure exhibits a high density of dislocations, which gives a good strength to the steel at room temperature. Many investigations have clearly shown that the steel is highly prone to cyclic softening [49 51]. In addition, The Low Cycle fatigue (LCF) experiments have shown a significant softening for the first hundred cycles following a linear continuous softening until the rupture. 

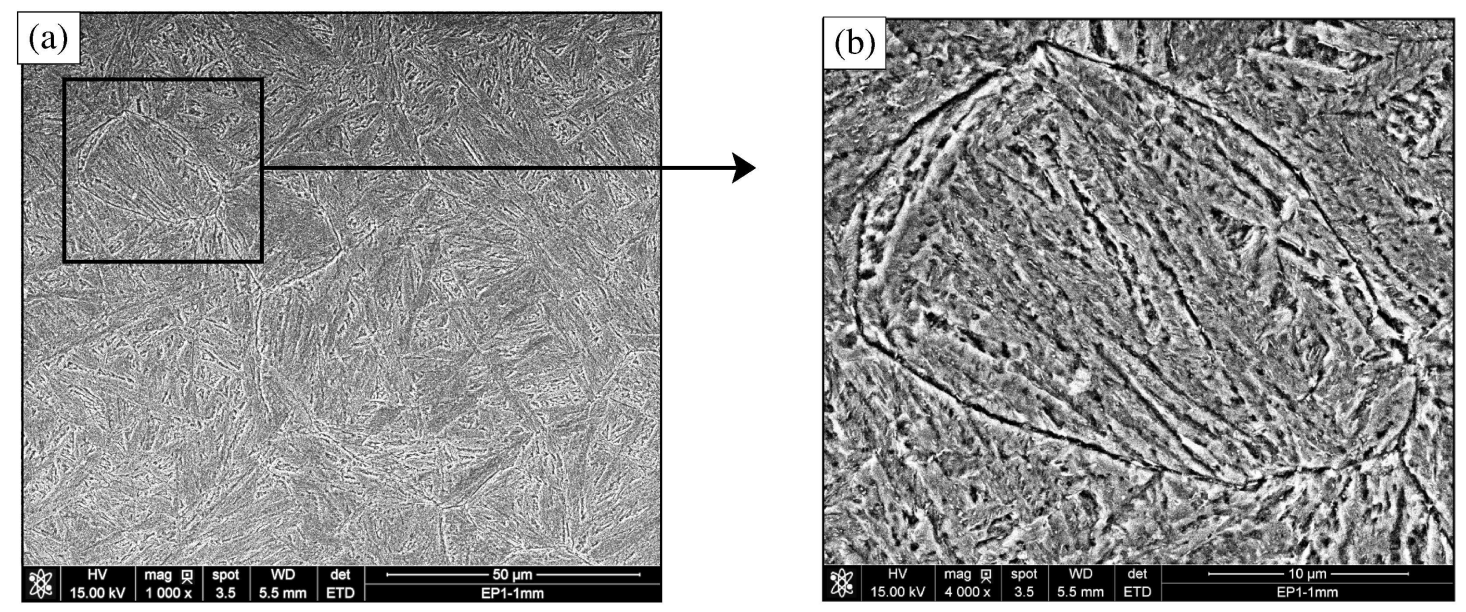

Figure 1: SEM observations of X38CrMoV5-1 steel: martensite lath microstructure (a) with prior austenitic grain (b).
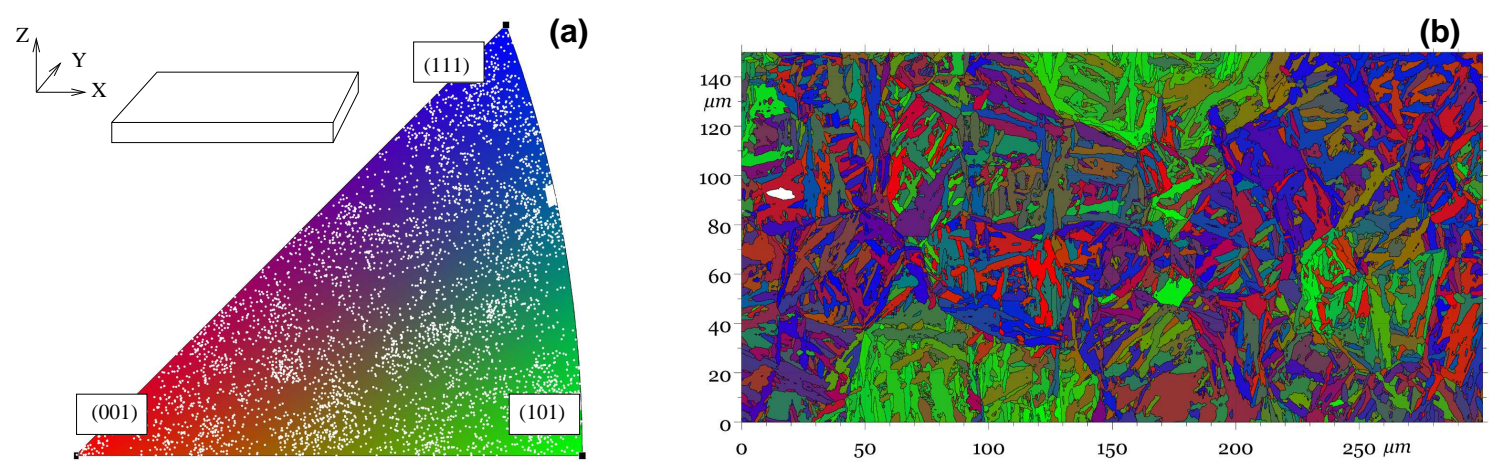

Figure 2: Inverse pole figure according $X$ axis, white dots correspond to the orientation of each martensitic lath (a); Spatial distribution of the orientations (b).

\subsection{Surface grain texturing}

The forging tool surface which can be defined as a layer between $10^{-2} \mathrm{~mm}$ and $10^{-6} \mathrm{~mm}$ constitutes a preferred zone for plasticity and damage mechanisms. This can be explained by various phenomena like wear, shear strain and thermomechanical fatigue in coupling with oxydation when tools are working at high temperature. In order to investigate the influence of surface and subsurface properties on the mechanical behavior of X38CrMoV5-1 steel, a servo-hydraulic tensile test machine, with a nominal force of $20 \mathrm{kN}$ which was previously adapted to fretting fatigue tests, is used [52]. It is combined with a secondary axis with a nominal force of $25 \mathrm{kN}$ allowing to apply a transverse compressive loading to the sample by the use of cylindrical pins. A back and forth axial displacement $u$ controlled test is combined with a transverse loading/unloading force $F$ as illustrated in Fig. 3. Hence, a surface with microstructural properties similar to those observed at the die surface during forging is obtained.

Such subsurface/surface mechanical and microstructural characteristics are achieved by applying high levels of loading. A limited number of cycles is enough to promote 


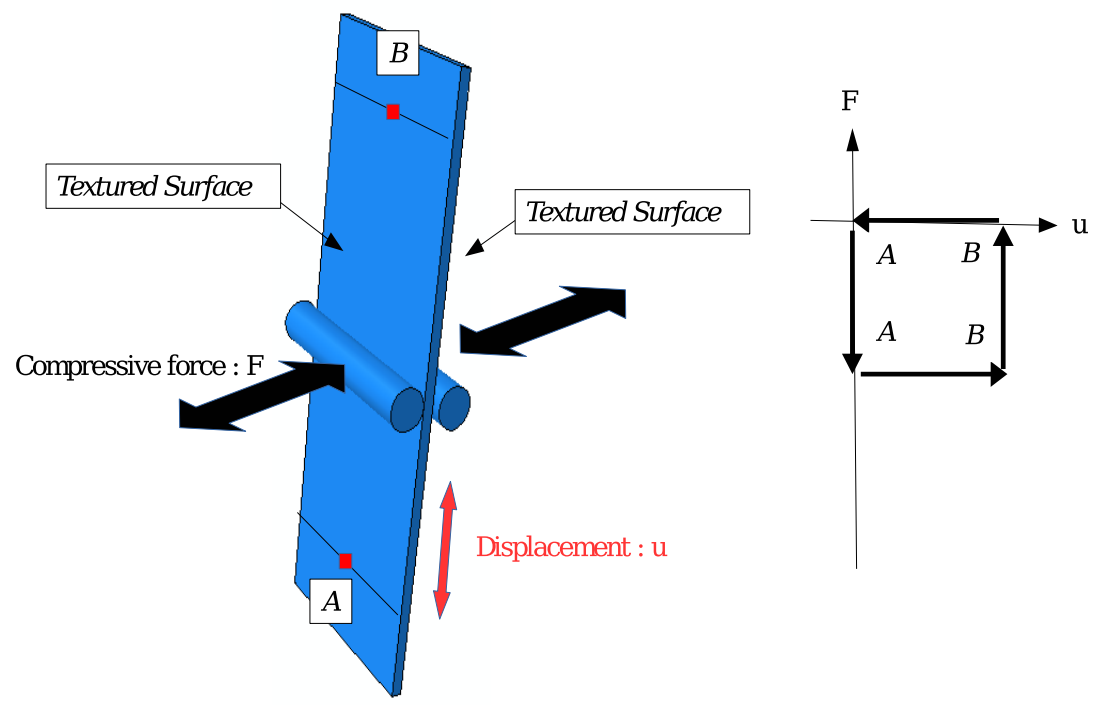

Figure 3: Experimental conditions of the surface generation process. Remark: the specimen is only hold by the movable actuator at one of its edges while the other is totally free to move.

inelastic flow. The tests use rectangular samples (270 $\mathrm{mm}$ long and $20 \mathrm{~mm}$ wide) with $1 \mathrm{~mm}$ thickness. Fig. 4 illustrates the SEM observation of the surface. In this case, 22 cycles of loading (forth axial displacement $u$ from $A$ to $B$ ) / unloading (back axial displacement $u$ from $B$ to $A$ ) are performed as shown in Fig. 3 , with a maximal force of $F=9.8 \mathrm{kN}$. EBSD analysis of a selected area, with a frame of $370 \times 270 \mu \mathrm{m}^{2}$ shows a significant gradient of the morphological texture, in-depth from the surface. In such zones, it is very difficult to identify a consistent set of Kikuchi lines for properly indexing the highly elongated grains. Both SEM and EBSD investigations have revealed a mechanically affected zone of about $65 \mu \mathrm{m}$.
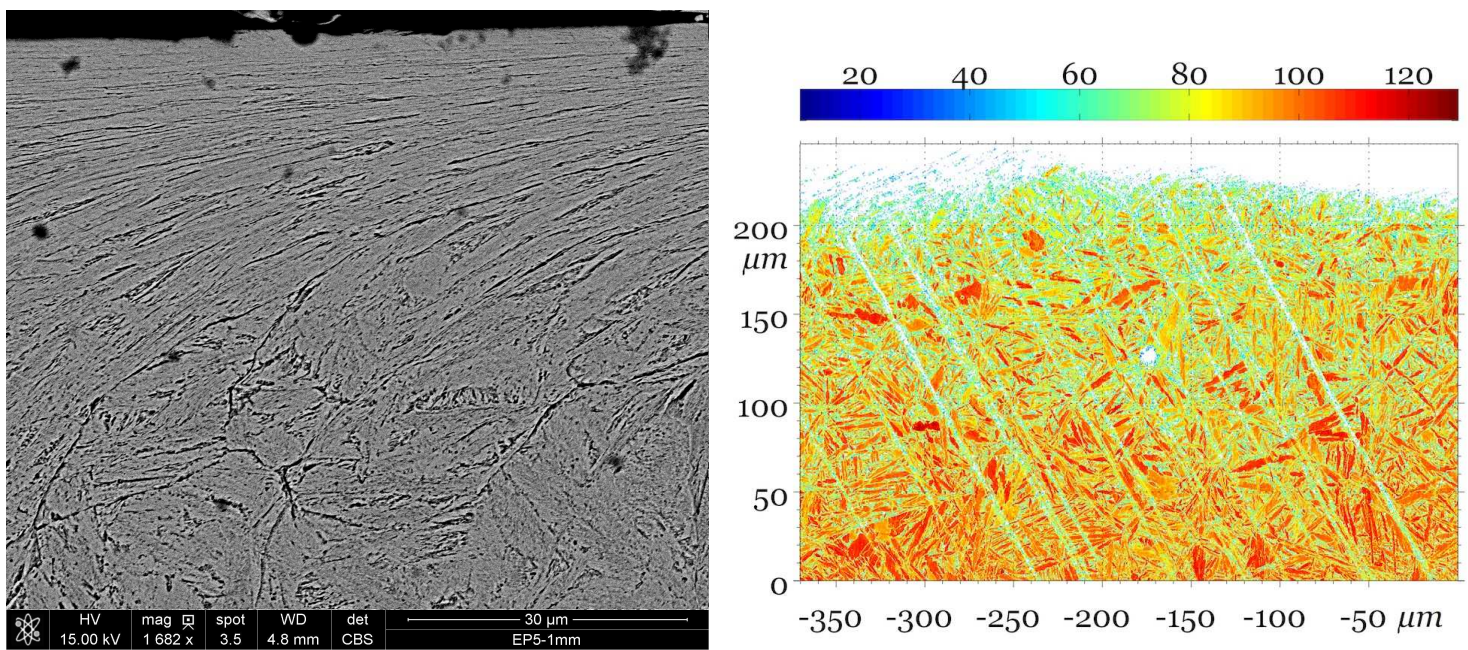

Figure 4: Surface analysis by SEM Observations and EBSD measurements 


\subsection{In situ analysis of the local behavior}

The specimen are machined from the previous sheet by Electrical Discharge Machining (EDM) such that the affected zone is kept on the gauge length as illustrated in Fig. 5. The tensile tests are conducted on a Instron servo-electric testing machine with a nominal force of $30 \mathrm{kN}$. The local strain measurement is done by using a Keyence optical microscope with a magnification factor of 1000. It is placed on a $x-y-z$ moving table for a very high accurate positioning of the microscope lens. An Area of Interest (AoI) of about $500 \times 500 \mu \mathrm{m}^{2}$ is selected for in-situ measurements during tensile loading. Digital images of $1600 \times 1200$ pixels are captured. The martensitic microstructure is used as a natural speckle pattern. Moreover, the global strain is measured by a classical MTS extensometer with a gauge length of $12.5 \mathrm{~mm}$. It is placed beside the AoI (see Fig. 5). It should be emphasized that both sides of the specimen after EDM and the highly deformed surface (see Fig. 5) are slightly polished to reduce the eventual surface cracks. Care is taken to avoid and eliminate totally the textured surface.

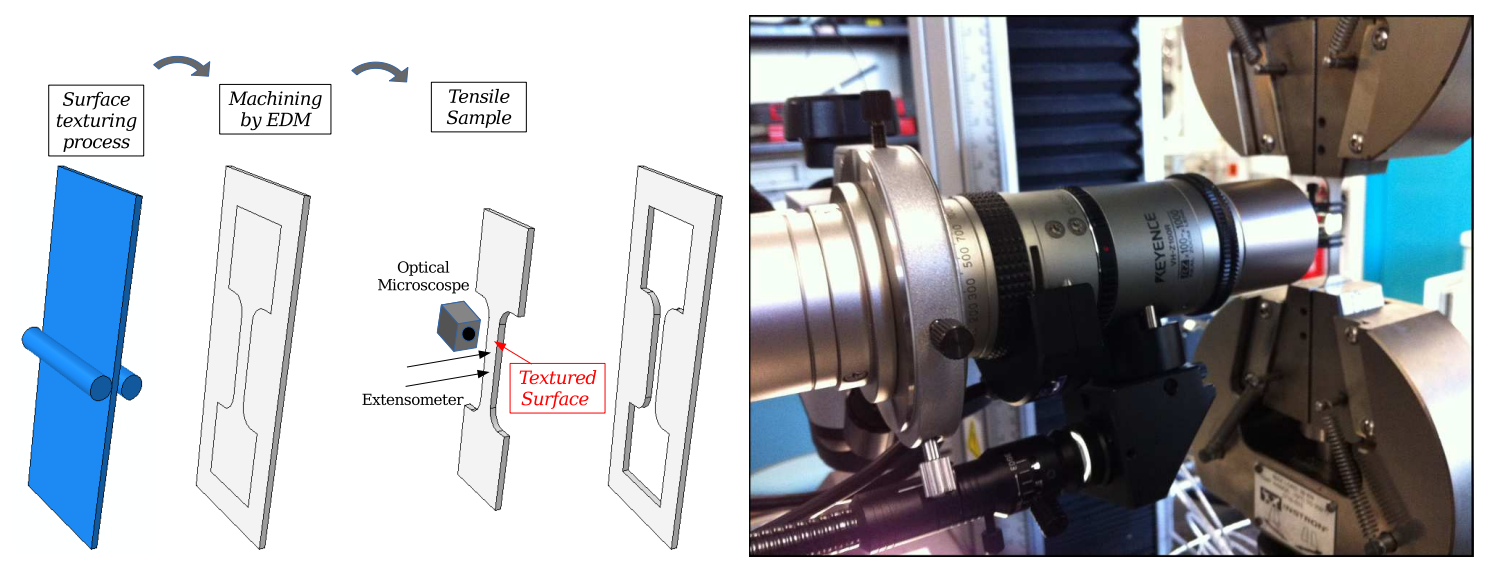

Figure 5: In-situ analysis of the deformation maps under monotonic loading by using Digital Image Correlation (DIC) technique

\subsection{RVE definition}

These measurements are used to assess the size of the RVE adapted to the X38CrMoV5-1 microstructure [53]. A preliminary tensile test is performed on a sample having a surface without any prior texturing. A tensile ductility of $8.5 \%$ is obtained with the global extensometer measurement. Several AoI are defined: $400 \times 400 \mu m^{2}\left(Z_{1}\right), 300 \times 300 \mu m^{2}\left(Z_{2}\right), 200 \times 200 \mu m^{2}\left(Z_{3}\right), 150 \times 150 \mu m^{2}\left(Z_{4}\right)$ and $100 \times 100 \mu \mathrm{m}^{2}\left(Z_{5}\right)$. A mean spatial strain field of these AoI is calculated and compared with the global strain measured by the extensometer. Based on the mean strain calculated for each AoI, it is concluded that the Zone 5 underestimates the global strain measured by extensometer. On the contrary, Zones 3 and 4 can be considered as a RVE since the mean strain obtained is similar to the global strain (see Fig. 6). The relative local ductility given by the equation $\delta=\left(A_{Z}^{\text {global }}-A_{Z}^{\text {AoI }}\right)$ is reported in Table 2 where $A_{Z}^{\text {AoI }}$ is the ductility calculated in each AoI and $A_{Z}^{\text {global }}$ is the global ductility. 

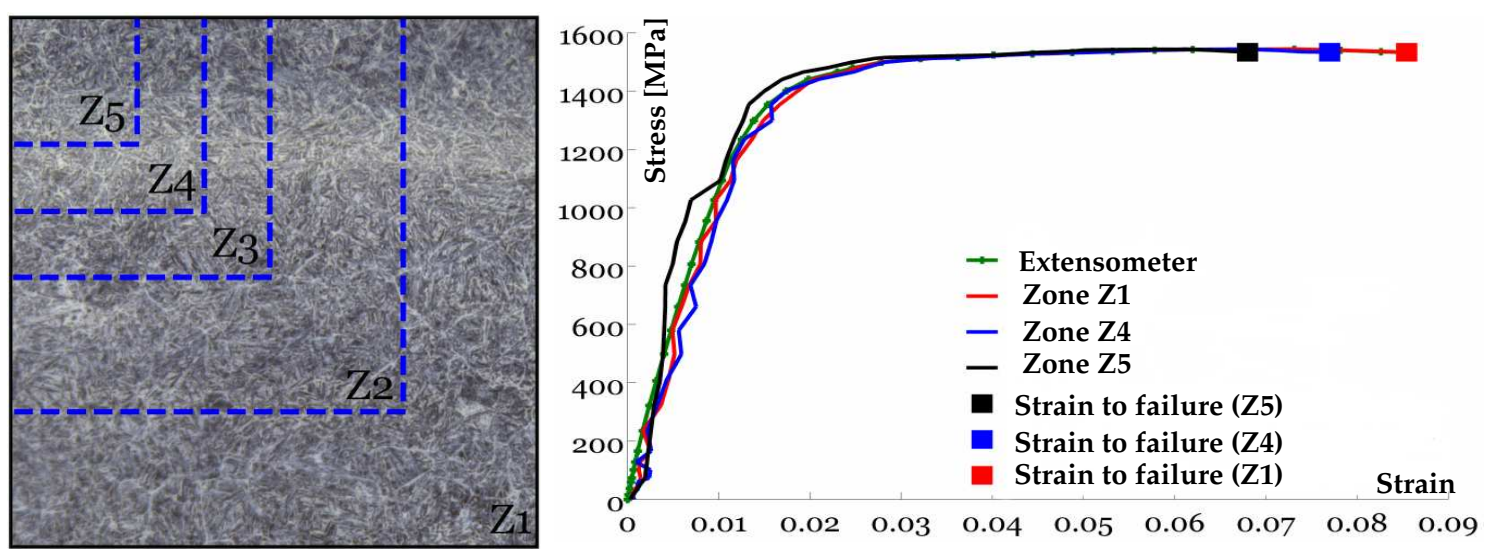

Figure 6: Estimation of the RVE size by using DIC technique and by comparison with the strain provided by the extensometer

Table 2: Gaps between the deformation to failure given by the extensomter and for each considered AoI

\begin{tabular}{cccccc}
\hline AoI & $Z_{1}$ & $Z_{2}$ & $Z_{3}$ & $Z_{4}$ & $Z_{5}$ \\
\hline$\delta[\%]$ & +3 & +3 & +3 & -9.41 & -20.09 \\
\hline
\end{tabular}

\subsection{Tensile tests conducted on textured surface}

As an example, in Fig. 7, three strain fields are shown at various macroscopic strain levels $(0.5 \% ; 1 \% ; 3 \%)$. The major strain field evolution is analyzed for each image. For a global $0.5 \%$ elastic strain, a local major strain of $2.5 \%$ is calcuated by DIC. Thus, a local plastic behavior occurs even if the macroscopic behavior remains elastic. Deformation seems to be initiated at the PAG boundaries and also is dependent on their orientations. At a global strain level of $1 \%$ and $3 \%$, the strain is significant and seems to be localized at $\pm 45^{\circ}$ with respect to the tensile axis. Previous investigations performed on X38CrMoV5-1 tool steel [54] have shown an important plastic flow at the interface between the textured surface/subsurface and the bulk. This plasticity is localized at the boundaries of PAG and is responsible for micro voids initiation. This phenomenon causes the failure by decohesion at PAG and lath boundaries. It can explain the damage initiation at the subsurface of the material. The local strain field with the band at $\pm 45^{\circ}$ may be related to this mechanism. 

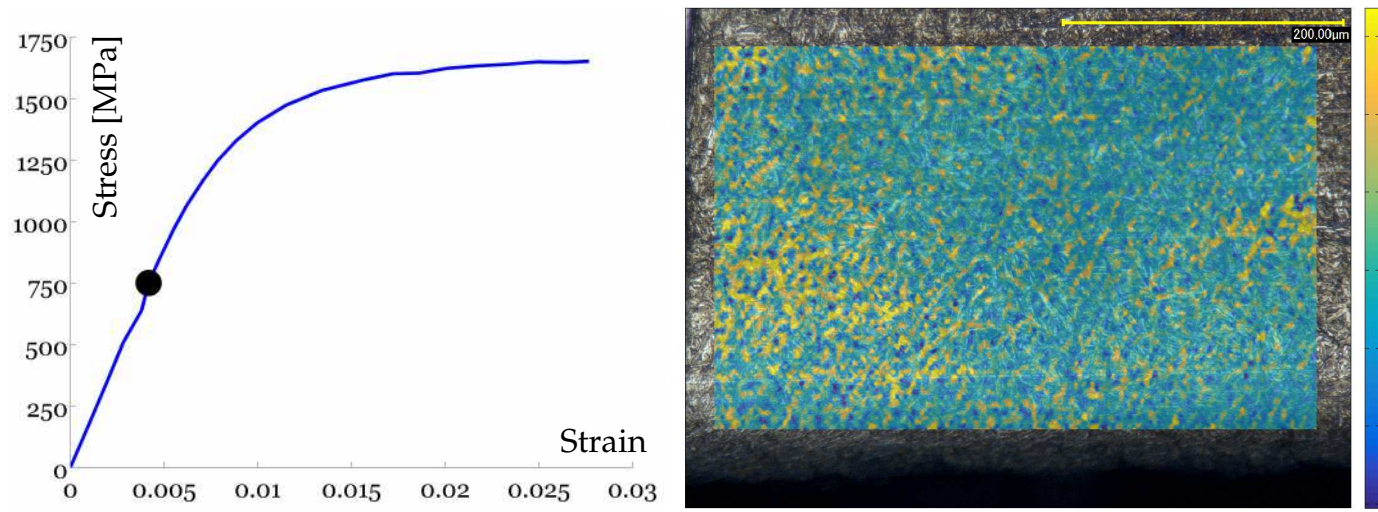

0.025

0.019

0.013

0.008

0.002

$-0.004$

$-0.01$
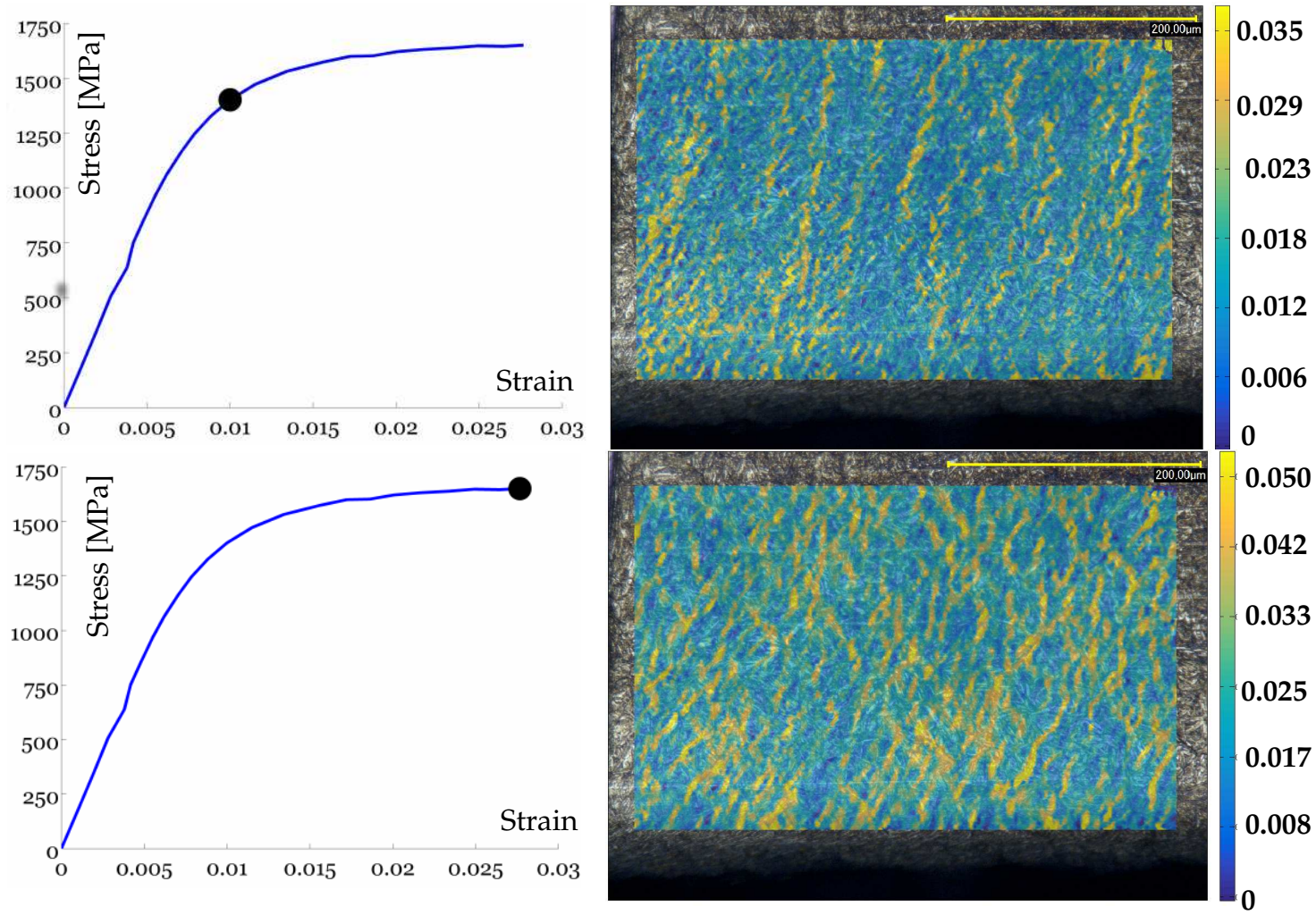

Figure 7: Major stain measurements provided by DIC technique at different macroscopic deformations $(0.5 \% ; 1 \% ; 3 \%)$ 


\section{Multi-scale behavior modeling}

In the present investigation, the multi-scale approach is based on the behavior model proposed by Meric and Cailletaud [34, 35], this approach satisfies the requirements of the thermodynamics of irreversible processes [6, 23]. In this theoretical framework, the state laws or thermodynamic potentials can be written at different scales. The local behavior equations are formulated at the microscopic scale considering the slip systems. The macroscopic RVE, previously defined, corresponds to a volume of $150 \times 150 \times 50 \mu \mathrm{m}^{3}$. Inbetween these two scales, a mesoscopic scale corresponding to a martensitic lath is assumed. The martensitic lath blocks in a PAG are defined as an intermediate scale between the microscopic and the macroscopic scales. This micro-meso-macro approaches leads to define a multi-scale modeling and are governed by scale transition rules presented hereafter. Many works in the multi scale approaches using crystal plasticity modeling employ a finite strain (large strain) formulation [55, 56]. Nevertheless, bodies in relative movements (forging tool or pin on disc testing ...) are subjected to cumulative plastic straining with small cyclic strain ranges achieving definitively to high strain levels. Therefore, in the present study, a modeling framework based on small strains assumption is addressed. However, a finite strain approach is carried out and reported in [37].

\subsection{Constitutive Equations}

The Helmholtz potential energy density per unit mass usually defined at the macroscopic scale can also be considered as a potential given as the sum of free energies at the mesoscopic scale.

$$
\varrho \Psi=\varrho \Psi_{e}\left(\left\langle\underset{\sim}{\varepsilon_{\boldsymbol{e}}}\right\rangle_{\mathcal{V}}\right)+\varrho \Psi_{i n}\left(q_{i}\right)=\sum_{i=1}^{N}\left(\underset{\sim}{\varrho} \Psi_{e}^{i}\left(\boldsymbol{\varepsilon}_{\boldsymbol{e}}\right)+\varrho \Psi_{i n}^{i}\left(\rho_{s}, \alpha_{s}\right)\right)
$$

where $\varrho$ is the material density, $N$ is the lath number in $\mathcal{V},\left\langle\underset{\sim}{\varepsilon_{\boldsymbol{e}}}\right\rangle_{\mathcal{V}}$ the average elastic strain tensor in $\mathcal{V}$ and $q_{i}$ the internal variables including the isotropic $\rho_{s}$ and kinematic $\alpha_{s}$ hardening variables resolved on each slip system $s$.

As mentioned earlier, small deformation conditions and an additive strain partition $\underset{\sim}{\boldsymbol{\varepsilon}}=\boldsymbol{\varepsilon}_{\boldsymbol{e}}+\boldsymbol{\varepsilon}_{\boldsymbol{i n}}$ are assumed. Moreover, a linear isotropic behaviour is considered. Therefore, the elasticity tensor is described by two parameters that are macroscopic Young modulus $E$ and Poisson ratio $\nu$.

In Eq. 1, the non linear Helmholtz free energy $\Psi_{i n}^{i}$, for a given lath $i$, is associated to the non linear part of the behavior at the mesoscopic scale by considering the hardening variables formulated at a microscopic scale.

$$
\varrho \Psi_{i n}^{i}\left(\rho^{s}, \alpha^{s}\right)=\frac{1}{2} C \sum_{s=1}^{N_{s}} \alpha^{s 2}+\frac{1}{2} b Q \sum_{s=1}^{N_{s}} \sum_{r=1}^{N_{s}} h^{s r} \rho^{s} \rho^{r}
$$

$C$ and $Q$ are material parameters related to kinematic and isotropic hardening respectively. $b$ is the isotropic hardening rate parameter and $N_{s}$ is the number of slip systems potentially activated. For BCC crystal, the number of slip systems is given by slip planes $\left\{\begin{array}{lll}1 & 1 & 0\end{array}\right\},\left\{\begin{array}{lll}1 & 1 & 2\end{array}\right\},\left\{\begin{array}{lll}1 & 2 & 3\end{array}\right\}$ and slip directions $\left\langle\begin{array}{llll}1 & 1 & 1\end{array}\right\rangle$. It confers 
to the polycrystal 48 activable slip systems. In the present study, the deformation mechanisms related to easy glide and pencil glide mechanisms are considered to be predominant, e.g: $\{110\}\langle 111\rangle$ and $\{112\}\langle 111\rangle$ [57]. Therefore, the number of active slip systems is reduced and only $24 \times 24$ interaction matrix $\boldsymbol{h}$ including 8 coefficients $h_{i}$ symmetrically allocated [8, 58, 59] is required (Eq. A.1 into the appendix section). The previous slip system families are given by the Schmid-Boas notations in Eq. A.1. The link between Miller indexes and Schmid-Boas system is provided in Tab. A.8 shown into the same appendix section. The macroscopic Cauchy stress $\underset{\sim}{\boldsymbol{\Sigma}}=\langle\underset{\sim}{\boldsymbol{\sigma}}\rangle_{\mathcal{V}}$ is the average stress tensor in $\mathcal{V}$ (Eq. 3), it is obtained by derivation of the Helmotz free energy (see Eq. 1 and Eq. 2).

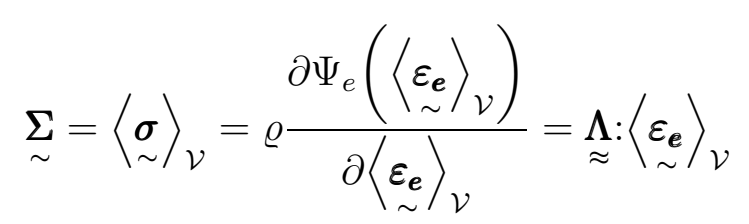

The associated variables related to the isotropic and kinematic hardening at the microscopic scale are described in Eq,4 and 5 ,

$$
\begin{aligned}
& r^{s}=\varrho \frac{\partial \Psi_{i n}^{i}\left(\rho^{s}, \alpha^{s}\right)}{\partial \rho^{s}}=b Q \sum_{r=1}^{N_{s}} h^{s r} \rho^{r} \\
& \chi^{s}=\varrho \frac{\partial \Psi_{i n}^{i}\left(\rho^{s}, \alpha^{s}\right)}{\partial \alpha^{s}}=C \alpha^{s}
\end{aligned}
$$

Similarly, the evolution equations are obtained at the macroscopic scale by defining a viscoplastic potential $\Omega$ at the mesoscopic scale. This global potential being the sum of the partial potentials $\Omega_{s}$ obtained at the microscopic scale (Eq. 6).

$$
\Omega=\sum_{s=1}^{N_{s}} \Omega_{s}=\sum_{s=1}^{N_{s}} \frac{K}{n+1}\left\langle\frac{f^{s}}{K}\right\rangle^{n+1}
$$

where $K$ and $n$ are parameters related to the material viscosity and $f^{s}$ is the yield function of the slip system $s$ given by Eq. 7 .

$$
f^{s}=\left|\tau^{s}-\chi^{s}\right|-r^{s}-\tau_{0}^{s}
$$

where $\tau^{s}$ and $\tau_{0}^{s}$ are respectively the resolved shear stress and the critical resolved shear stress.

This approach can be classified in the frame of non associated models [6] since it is defined by two functions, the function $f$ (Eq. 7) for the elasticity domain and the flow potential given by $F$ (Eq. 8).

$$
F^{s}=f^{s}+b \rho^{s} r^{s}+d \alpha^{s} \chi^{s}
$$

where $d$ is a material parameter related to the kinematic variable. 
From Eq. 6] and 8, the evolution equations of internal variables (Eq. 9.11) are obtained as:

$$
\begin{aligned}
& \dot{\gamma}^{s}=\dot{\lambda}^{s} \frac{\partial F^{s}}{\partial \tau^{s}}=\left\langle\frac{f^{s}}{K}\right\rangle^{n} \operatorname{sign}\left(\tau^{s}-\chi^{s}\right)=\dot{v}^{s} \operatorname{sign}\left(\tau^{s}-\chi^{s}\right) \\
& \dot{\rho}^{s}=-\dot{\lambda}^{s} \frac{\partial F^{s}}{\partial r^{s}}=\left(1-b \rho^{s}\right) \dot{v}^{s} \\
& \dot{\alpha}^{s}=-\dot{\lambda}^{s} \frac{\partial F^{s}}{\partial \chi^{s}}=\left(\operatorname{sign}\left(\tau^{s}-\chi^{s}\right)-d \alpha^{s}\right) \dot{v}^{s}
\end{aligned}
$$

where $\dot{\lambda}^{s}$ is a viscoplastic multiplier which is derivated from the viscoplastic potential $\Omega_{s}$ at the microscopic scale (Eq. 12)

$$
\dot{\lambda}^{s}=\frac{\partial \Omega_{s}}{\partial f^{s}}=\dot{v}^{s}
$$

The Schmid law (Eq. 13) and the yield function (Eq. 7) define the mesoscopic non linear strain rate (Eq. 14).

$$
\begin{gathered}
\tau^{s}=\underset{\sim}{\sigma}: \operatorname{sym}\left(\underline{l}^{s} \otimes \underline{n}^{s}\right) \\
{\underset{\sim}{\dot{\varepsilon}}}^{i n}=\frac{\partial \Omega}{\partial \underset{\sim}{\sigma}}=\sum_{s=1}^{N_{s}} \frac{\partial \Omega_{s}}{\partial \underset{\sim}{\sigma}}=\sum_{s=1}^{N_{s}} \dot{\lambda}^{s} \frac{\partial f^{s}}{\partial \underset{\sim}{\sigma}}=\sum_{s=1}^{N_{s}} \dot{\gamma}^{s} \operatorname{sym}\left(\underline{n}^{s} \otimes \underline{l}^{s}\right)
\end{gathered}
$$

where $\underline{n}^{s}$ is the slip plane normal vector and $\underline{l}^{s}$ the slip direction in this plane.

The state laws (Eq. 4 and 5) and the evolution equations (Eq. 95.13) express the intrinsic dissipation $\Theta$ as:

$$
\begin{aligned}
\Theta & =\underset{\sim}{\sigma: \dot{\varepsilon}_{\sim}^{i n}}-\sum_{s=1}^{N_{s}} \chi^{s} \dot{\alpha}^{s}-\sum_{s=1}^{N_{s}} r^{s} \dot{\rho}^{s} \\
& =\sum_{s=1}^{N_{s}}\left(\tau^{s} \dot{\gamma}^{s}-\chi^{s}\left(\operatorname{sign}\left(\tau^{s}-\chi^{s}\right)-d \alpha^{s}\right) \dot{v}^{s}-r^{s}\left(1-b \rho^{s}\right) \dot{v}^{s}\right) \\
& =\sum_{s=1}^{N_{s}}\left(f^{s}+\tau_{0}^{s}+\frac{d}{C}\left(\chi^{s}\right)^{2}+b r^{s} \rho^{s}\right)
\end{aligned}
$$

Table 3 summarizes the equations set.

\subsection{Strategies for identification of the model parameters}

As observed in the above, DIC measurements reveal heterogeneous strain fields distribution in tensile tests. Therefore, a straightforward identification of the material paramters associated to the chosen model is impossible. Indeed a full field approach requires the generation of the virtual microstructure associated with the 
Table 3: Constitutive equations of the model of Meric and Cailletaud

\begin{tabular}{|c|c|c|}
\hline Strain decomposition & $\varepsilon=\varepsilon^{e}+\varepsilon^{i n}$ & \\
\hline Schmid law & $\tau_{s}=\boldsymbol{\sigma}: \operatorname{sym}\left(\underline{l}^{s} \otimes \underline{n}^{s}\right)$ & \\
\hline Microscopic flow rule & $\dot{v}^{s}=\left\langle\frac{\left|\tau^{s}-\chi^{s}\right|-r^{s}-\tau_{0}^{s}}{K}\right\rangle^{n}$ & $\dot{\gamma}^{s}=\dot{v}^{s} \operatorname{sign}\left(\tau^{s}-\chi^{s}\right)$ \\
\hline Mesoscopic flow rule & $\dot{\dot{\varepsilon}}^{i n}=\sum_{s=1}^{N_{s}} \dot{\gamma}^{s} \operatorname{sym}\left(\underline{n}^{s} \otimes \underline{l}^{s}\right)$ & \\
\hline $\begin{array}{l}\text { Kinematic hardening } \\
\text { Isotropic Hardening } \\
\text { Intrinsic dissipation }\end{array}$ & $\begin{array}{c}\chi^{s}=C \alpha^{s} \\
r^{s}=b Q \sum_{r=1}^{N_{s}} h^{s r} \rho^{r} \\
\Theta=\underset{\sim}{\sigma} \dot{\varepsilon}^{i n}-\sum_{s=1}^{N_{s}} \chi^{s} \dot{\alpha}^{s}-\sum_{s=1}^{N_{s}} r^{s} \dot{\rho}^{s}\end{array}$ & $\begin{array}{l}\dot{\alpha}^{s}=\dot{\gamma}^{s}-d \alpha^{s} \dot{v}^{s} \\
\dot{\rho}^{s}=\left(1-b \rho^{s}\right) \dot{v}^{s}\end{array}$ \\
\hline
\end{tabular}

knowledge of various slip systems and crystallographic orientations. In addition, the mechanical tests should be combined with high resolution EBSD measurements. Consequently, the optimisation method becomes very time consuming which lead to consider a mean field approach. As mentionned previously, the Berveiller-Zaoui model [18] and $\beta$-model of Pilvin and Cailletaud [10, 19] are combined for the parameters identification purpose. This latter is relevant for modelling the behavior of softening materials as it is observed during the cyclic behavior of hot work tool steels [60]. For Berveiller-Zaoui model, the localization rule of the stress tensor is given by Eq. 16.

$$
\begin{array}{r}
\boldsymbol{\sigma}_{\sim}^{\varphi}=\underset{\sim}{\boldsymbol{\Sigma}}+2 \mu \alpha(1-\beta):\left(\underset{\sim}{\left.\boldsymbol{E}^{p}-\underset{\sim}{\boldsymbol{\varepsilon}^{\varphi, p}}\right)}\right. \\
\text { with: } \frac{1}{\alpha}=1+\frac{3}{2} \mu \frac{E_{e q}^{p}}{\Sigma_{e q}} \quad \text { and: } \beta=\frac{2(4-5 \nu)}{15(1-\nu)}
\end{array}
$$

where $\boldsymbol{\sigma}_{\sim}^{\varphi}$ and $\underset{\sim}{\boldsymbol{\Sigma}}$ are respectively the stresses at the mesoscopic/lath and macroscopic scales, $\nu$ is the Poisson ratio, $\mu$ the shear modulus and $\alpha$ stands for a non linear accommodation parameter whose formulation is a function of the von Mises equivalent inelastic strain $E_{e q}^{p}$ and stress $\Sigma_{e q}$ at the macroscopic scale.

In $\beta$-model (Eq. 17), the transition is given as a difference between a global $\underset{\sim}{\boldsymbol{B}}$ and local $\beta^{\varphi}$ variables in order to describe the non linear accommodation whereas the Berveiller-Zaoui model considers a difference between a global $\underset{\sim}{\boldsymbol{E}^{p}}$ and local $\underset{\sim}{\boldsymbol{\varepsilon}^{\varphi, p}}$ inelastic strains.

$$
\begin{array}{r}
\underset{\sim}{\boldsymbol{\sigma}^{\varphi}}=\underset{\sim}{\boldsymbol{\Sigma}}+2 \mu(1-\beta):\left(\underset{\sim}{\boldsymbol{B}-\boldsymbol{\beta}^{\varphi}}\right) \\
\text { with: } \underset{\sim}{\boldsymbol{B}}=\left\langle\underset{\sim}{\boldsymbol{\beta}^{\varphi}}\right\rangle=\sum_{\varphi} \underset{\sim}{f_{\varphi} \boldsymbol{\beta}^{\varphi}}
\end{array}
$$

Moreover, $\beta^{\varphi}$ presents a non linear evolution with respest to inelastic strain. This evolution law (Eq. 18) can be written under a Armstrong-Frederick form [5, [6]. Thus, this transition rule can be extended to complex loading paths like cyclic behavior. 


$$
\underset{\sim}{\dot{\boldsymbol{\beta}}^{\varphi}}=\underset{\sim}{\dot{\boldsymbol{\varepsilon}}^{p, \varphi}}-\underset{\sim}{D \boldsymbol{\beta}^{\varphi}} \dot{\varepsilon}_{e q}^{p, \varphi}
$$

where $\dot{\varepsilon}_{e q}^{p, \varphi}$ is the equivalent inelastic strain rate at the mesoscopic scale. The $\beta$-model is reliable for both monotonic and cyclic loadings but requires the identification of an additional coefficient $D$.

The parameters identification is based on experimental tensile and cyclic straincontrolled tests at room temperature. A servo-hydraulic testing machine and Testar $2 \mathrm{~S}$ controller are used. The push-pull tests with a strain rate of $10^{-2} s^{-1}$ exhibit a cyclic softening from the first cycle to the failure which is a well known behavior of martensitic hot work tool steels [50, 61, 62]. Several strain ranges are examined under two strain ratios $R_{\varepsilon}=\{-1,0\}$ (Table 41).

Table 4: Tensile and cyclic test conditions, $\dot{\varepsilon}=10^{-2} \mathrm{~s}^{-1}$

\begin{tabular}{lccccc}
\hline Test number & tensile 1 & cyclic 1 & cyclic 2 & cyclic 3 & cyclic 4 \\
\hline Strain range & $0-10 \%$ & $\pm 0.8 \%$ & $\pm 0.9 \%$ & $\pm 1.1 \%$ & $0-1.5 \%$ \\
\hline
\end{tabular}

The identification process of the model parameters contains several steps. First, by assuming an isotropic behavior, the elasticity tensor $\underset{\approx}{\boldsymbol{\alpha}}$ is identified using the Young modulus $E$ and the Poisson ratio $\nu$. As at room temperature, the material is not sensitive to strain rate, the viscous parameters $K$ and $n$ are set to provide a rate-independent model response which can be considered as a limiting case of classical viscoplasticity. The kinematic and isotropic hardening coefficients and the transition rule parameter are identified from experimental tests. For this purpose, the following sequential stages (simulation and optimisation) are undertaken using the Zset solution software [63]:

- transition rule parameter, kinematic hardening and critical resolved shear stress are identified using the tensile curve,

- then the values of the kinematic hardening coefficients are refined using the stabilized cycles of the different cyclic tests (see table 4),

- isotropic hardening parameters are identified by fitting the both tensile and cyclic experiments,

- the interaction matrix coefficients are identified using the tensile test. In this case, the following constraints (see Eq. 19) are assumed according to the work of Hoc and Forest for BCC crystals [59]:

$$
h_{8} \leq h_{2} \leq h_{3} \leq h_{5} \leq h_{4} \leq h_{1} \leq h_{6} \leq h_{7}
$$

The interactions between slip systems $\{110\}\langle 111\rangle$ and $\{112\}\langle 111\rangle$ are classified in different types. They can belong to the same system ( $h 1$ and $\left.h_{8}\right)$, to colinear system $\left(h_{2}, h_{3}\right.$ and $\left.h_{6}\right)$ or to non-colinear system $\left(h_{5}, h_{4}\right.$ and $\left.h_{7}\right)$. Interactions between same or colinear slip systems of the family $\left\{\begin{array}{ll}1 & 10\end{array}\right\}$ 
Table 5: Material parameters

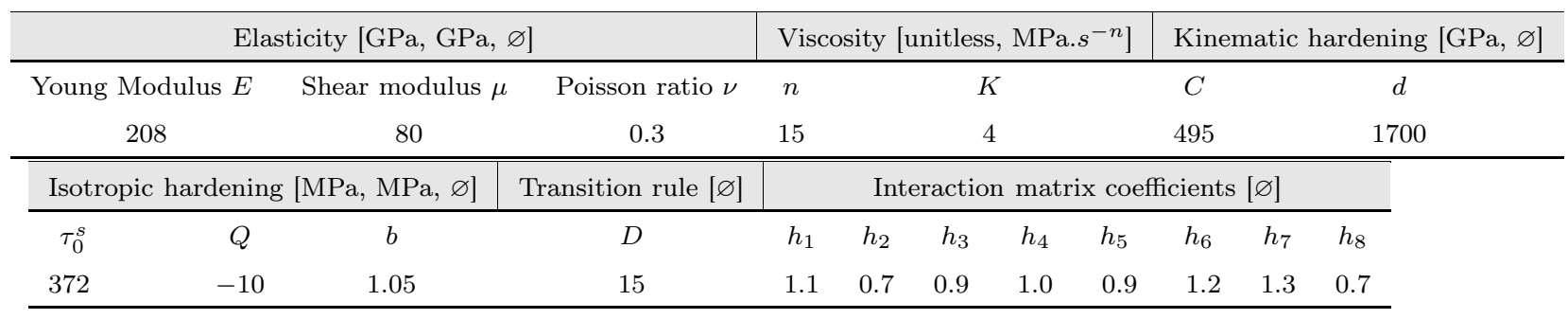

327

$\langle 111\rangle$ are assumed smaller than interactions between colinear slip systems not belonging to the same family, this latter being smaller than interactions between non colinear slip systems itself smaller than interactions between non colinear system of the family $\{110\}\langle 111\rangle$. Lastly, interactions between slip systems of the family $\left\{\begin{array}{lll}1 & 1\end{array}\right\}\langle 111\rangle$ are assumed greater than interactions between slip system of the family $\{110\}\langle 111\rangle$.

- Model validation by simulation of the tensile test using the transition rule of Berveiller-Zaoui.

The results of the identification process are illustrated in Table 5, The comparison between the results provided by the Meric-Cailletaud model using the $\beta$-model transition rule and our experiments are given for cyclic and tensile loading paths (Fig. 8)
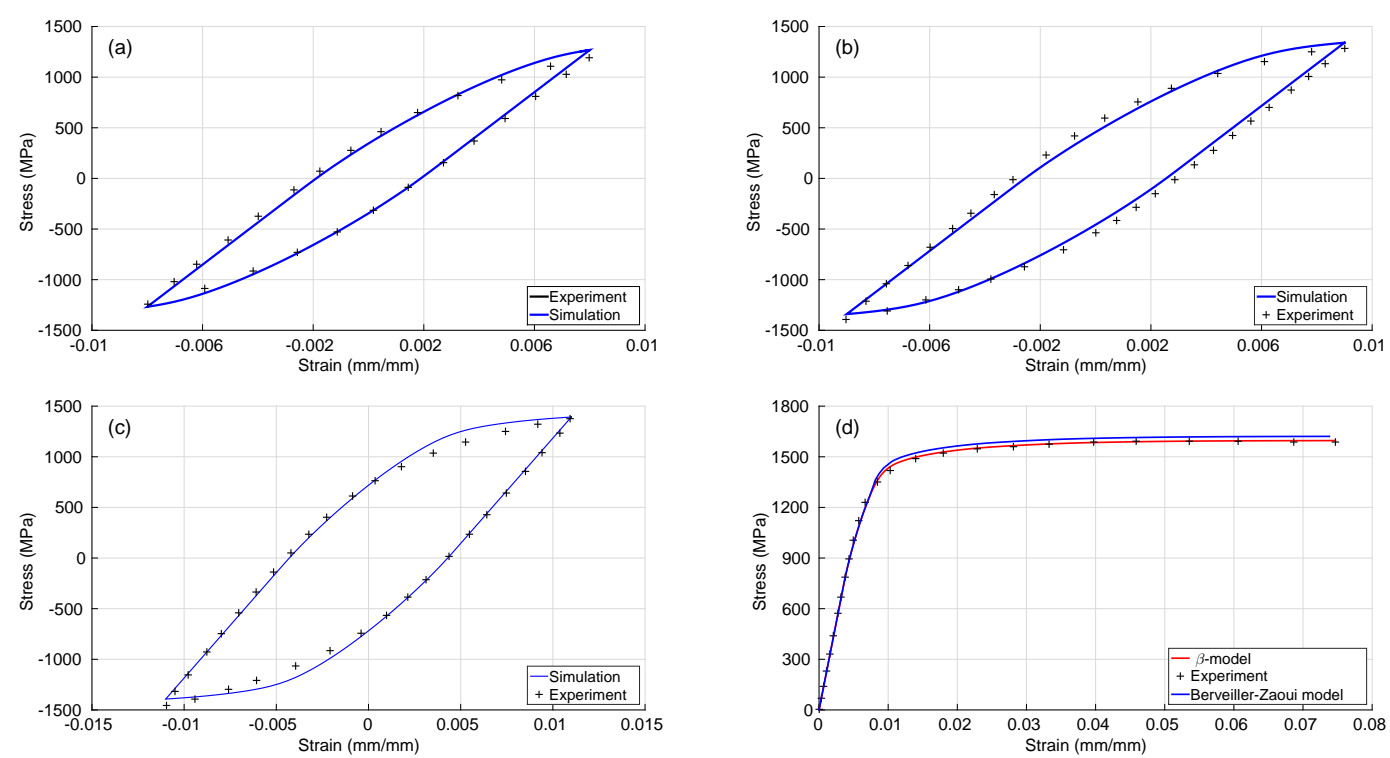

Figure 8: Comparison between computed strain-stress curve provided and experiment for the stabilized cycle (a-c); test cyclic1 (a); test cyclic2 (b); test cyclic3 (c); and for test tensile1 using Berveiller-Zaoui and $\beta$ models (d) 


\section{Numerical simulations}

\subsection{Finite Element implementation of the constitutive equations}

A lot of investigations deals with the FE implementation of a multiscale approach [4, 64 69]. In the present work, the rate tangent modulus method [15, 16] is used to implement the multiscale constitutive equations. The method consists in a direct resolution scheme and is based on a predictor/corrector algorithm of Simo and Hughes [70] where the Cauchy stress $\boldsymbol{\sigma}$ is written at the mesoscopic scale.

$$
\underset{\sim}{\sigma}=\underset{\approx}{\Lambda}:\left(\underset{\sim}{\varepsilon}-\underset{\sim}{\varepsilon^{i n}}\right)
$$

From Eq. 20 and using Eq. 14, Eq. 21 is obtained.

$$
\underset{\sim}{\dot{\sigma}}=\underset{\approx}{\Lambda}:\left(\underset{\sim}{\dot{\varepsilon}}-\sum_{s=1}^{N_{s}} \dot{\gamma}^{s} \operatorname{sym}\left(\underline{l}^{s} \otimes \underline{n}^{s}\right)\right)
$$

The incremental shear strain $\Delta \gamma^{s}$ on a prescribed system $s$ at time $t$ is defined by Eq. 22, where $\Delta t$ is the time increment.

$$
\Delta \gamma^{s}=\gamma_{t+\Delta t}^{s}-\gamma_{t}^{s}
$$

Interpolation versus time can be expressed as Eq. 23.

$$
\Delta \gamma^{s}=\left((1-\theta) \dot{\gamma}_{t}^{s}+\theta \dot{\gamma}_{t+\Delta t}^{s}\right) \Delta t
$$

By Taylor expansion of $\dot{\gamma}_{t+\Delta t}^{s}$, Eq. 24 then Eq. 25 can be achieved. In each slip system $s$, the shear strain rate $\dot{\gamma}^{s}$ is considered as the integration variable.

$$
\begin{aligned}
& \dot{\gamma}_{t+\Delta t}^{s}=\dot{\gamma}_{t}^{s}+\left.\frac{\partial \dot{\gamma}^{s}}{\partial \tau^{s}}\right|_{t} \Delta \tau^{s}+\left.\frac{\partial \dot{\gamma}^{s}}{\partial r^{s}}\right|_{t} \Delta r^{s}+\left.\frac{\partial \dot{\gamma}^{s}}{\partial \chi^{s}}\right|_{t} \Delta \chi^{s} \\
& \frac{\Delta \gamma^{s}}{\Delta t}=\dot{\gamma}_{t}^{s}+\left.\theta \frac{\partial \dot{\gamma}^{s}}{\partial \tau^{s}}\right|_{t} \Delta \tau^{s}+\left.\theta \frac{\partial \dot{\gamma}^{s}}{\partial r^{s}}\right|_{t} \Delta r^{s}+\left.\theta \frac{\partial \dot{\gamma}^{s}}{\partial \chi^{s}}\right|_{t} \Delta \chi^{s}
\end{aligned}
$$

where $\Delta \tau^{s}, \Delta r^{s}$ et $\Delta \chi^{s}$ are respectively the resolved shear stress, isotropic hardening and kinematic hardening increment acting on slip system $s$. These increments can be determined by using the constitutive equations (see Eq. 26, 27) and 28).

$$
\begin{aligned}
& \Delta \tau^{s}=\left(\underset{\approx}{\Lambda}: \operatorname{sym}\left(\underline{l}^{s} \otimes \underline{n}^{s}\right)\right):\left(\Delta \underset{\sim}{\varepsilon}-\sum_{r=1}^{N_{s}} \Delta \gamma^{r} \operatorname{sym}\left(\underline{l}^{r} \otimes \underline{n}^{r}\right)\right) \\
& \Delta r^{s}=b Q \sum_{r=1}^{N_{s}} h^{s r}\left(1-b \rho^{r}\right) \Delta \gamma^{r} \operatorname{sign}\left(\tau^{r}-\chi^{r}\right) \\
& \Delta \chi^{s}=C \Delta \gamma^{s}-C d \alpha^{s} \Delta \gamma^{s} \operatorname{sign}\left(\tau^{s}-\chi^{s}\right)
\end{aligned}
$$

Eq. 29 is obtained by introducing the previous equations into Eq. 25. 


$$
\begin{aligned}
\frac{\Delta \gamma^{s}}{\Delta t}=\dot{\gamma}_{t}^{s} & +\left.\theta \frac{\partial \dot{\gamma}^{s}}{\partial \tau^{s}}\right|_{t}\left(\underset{\approx}{\Lambda}: \operatorname{sym}\left(\underline{l}^{s} \otimes \underline{n}^{s}\right)\right):\left(\Delta \underset{\sim}{\varepsilon}-\sum_{r=1}^{N_{s}} \Delta \gamma^{r} \operatorname{sym}\left(\underline{l}^{r} \otimes \underline{n}^{r}\right)\right) \\
& +\left.\theta \frac{\partial \dot{\gamma}^{s}}{\partial r^{s}}\right|_{t}\left(b Q \sum_{r=1}^{N_{s}} h^{s r}\left(1-b \rho_{t}^{r}\right) \Delta \gamma^{r} \operatorname{sign}\left(\tau^{r}-\chi^{r}\right)\right) \\
& +\left.\theta \frac{\partial \dot{\gamma}^{s}}{\partial \chi^{s}}\right|_{t}\left(C \Delta \gamma^{s}-C d \alpha_{t}^{s} \Delta \gamma^{s} \operatorname{sign}\left(\tau^{s}-\chi^{s}\right)\right)
\end{aligned}
$$

341

342

Therefore, Eq. 29 can be expressed as a linear system (see Eq. 30) where the components of unknown vector $\Delta \gamma$ correspond to the shear strain increment for all slip systems.

$$
\underset{\sim}{A} \cdot \underline{\gamma}=\underline{b}
$$

with:

$$
\begin{aligned}
& \underset{\sim}{\boldsymbol{A}=A^{s r}=\delta^{s r}}+\left.\Delta t \theta \frac{\partial \dot{\gamma}^{s}}{\partial \tau^{s}}\right|_{t}\left(\underset{\approx}{\Lambda}: \operatorname{sym}\left(\underline{l}^{s} \otimes \underline{n}^{s}\right)\right):\left(\operatorname{sym}\left(\underline{l}^{r} \otimes \underline{n}^{r}\right)\right) \\
&-\left.\Delta t \theta \frac{\partial \dot{\gamma}^{s}}{\partial r^{s}}\right|_{t} b Q h^{s r}\left(1-b \rho^{r}\right) \operatorname{sign}\left(\tau^{s}-\chi^{s}\right) \\
&-\left.\Delta t \theta \frac{\partial \dot{\gamma}^{s}}{\partial \chi^{s}}\right|_{t}\left(\delta^{s r} C-\delta^{s r} C d \alpha^{s} \operatorname{sign}\left(\tau^{s}-\chi^{s}\right)\right) \\
& \underline{\Delta \boldsymbol{\gamma}}=\Delta \gamma^{r} ; \quad \underline{b}=b^{s}=\Delta t \dot{\gamma}^{s}+\left.\Delta t \theta \frac{\partial \dot{\gamma}^{s}}{\partial \tau^{s}}\right|_{t}\left(\underset{\approx}{\Lambda}: \operatorname{sym}\left(\underline{l}^{s} \otimes \underline{n}^{s}\right)\right): \Delta \underset{\sim}{\boldsymbol{\varepsilon}}
\end{aligned}
$$

$$
\begin{aligned}
& \left.\frac{\partial \dot{\gamma}^{s}}{\partial \tau^{s}}\right|_{t}=\frac{n}{K^{n}}\left(\left|\tau^{s}-\chi^{s}\right|-r^{s}-\tau_{0}^{s}\right)^{n-1} \\
& \left.\frac{\partial \dot{\gamma}^{s}}{\partial r^{s}}\right|_{t}=-\frac{n}{K^{n}}\left(\left|\tau^{s}-\chi^{s}\right|-r^{s}-\tau_{0}^{s}\right)^{n-1} \operatorname{sign}\left(\tau^{s}-\chi^{s}\right) \\
& \left.\frac{\partial \dot{\gamma}^{s}}{\partial \chi^{s}}\right|_{t}=-\frac{n}{K^{n}}\left(\left|\tau^{s}-\chi^{s}\right|-r^{s}-\tau_{0}^{s}\right)^{n-1}
\end{aligned}
$$

By solving the linear system given by Eq. 30, $\Delta \gamma^{s}$ is calculated and the shear strain rate $\dot{\gamma}^{s}=\frac{\Delta \gamma^{s}}{\Delta t}$ is assessed. As the rate tangent modulus method allows for a direct solving, the calculated shear strain increment may be not reliable when the time increment increases. Therefore this method is combined with a Newton Raphson resolution using the value provided by rate tangent modulus method as initial guess of the iterative algorithm. In the present investigation, an backward Euler or fully implicit method $(\theta=1)$ is assumed (Eq. 33) [71]. 


$$
\Delta \gamma^{s}-\Delta t \dot{\gamma}_{t+\Delta t}^{s}=0
$$

The numerical integration of the above constitutive equations is implemented on ABAQUS/Standard. As implicit solving of the equilibrium equations is used, the Jacobian matrix $\mathcal{J}=\frac{\partial \Delta \underset{\sim}{\tilde{\varepsilon}}}{\partial \Delta \underset{\sim}{\varepsilon}}$ associated to the nonlinear system is assessed from Eq. 21 (see Eq. 34).

$$
\begin{aligned}
\mathcal{J}=\frac{\partial \Delta \underset{\sim}{\boldsymbol{\sigma}}}{\partial \Delta \boldsymbol{\sim}} & =\frac{\partial\left[\underset{\sim}{\boldsymbol{\Lambda}}:\left(\Delta \underset{\sim}{\varepsilon}-\sum_{s=1}^{N_{s}} \Delta \gamma^{s} \operatorname{sym}\left(\underline{\boldsymbol{l}}^{s} \otimes \underline{\boldsymbol{n}}^{s}\right)\right)\right]}{\partial \Delta \underline{\sim}} \\
& =\underset{\approx}{\boldsymbol{\Lambda}} \underset{\approx}{\boldsymbol{\Lambda}}:\left[\sum_{s=1}^{N_{s}} \frac{\partial \Delta \gamma^{s}}{\partial \Delta \boldsymbol{\sim}} \otimes \operatorname{sym}\left(\underline{\boldsymbol{l}}^{s} \otimes \underline{\boldsymbol{n}}^{s}\right)\right]
\end{aligned}
$$

Hence, the term $\frac{\Delta \gamma^{s}}{\Delta \varepsilon}$ can be easily formulated by Eq. 30.

$$
\frac{\partial \Delta \gamma^{r}}{\partial \Delta \underset{\sim}{\varepsilon}}=\underset{\sim}{\boldsymbol{A}^{-1}} \cdot\left[\left.\Delta t \theta \frac{\partial \dot{\gamma}^{s}}{\partial \tau^{s}}\right|_{t} \underset{\sim}{\Lambda}: \operatorname{sym}\left(\underline{\boldsymbol{l}}^{s} \otimes \underline{\boldsymbol{n}}^{s}\right)\right]
$$

and by introducing Eq. 36 into Eq. 34, the final expression of $\mathcal{J}$ is given by Eq. 37 ,

$$
\mathcal{J}=\underset{\approx}{\boldsymbol{\Lambda}}-\left.\sum_{r=1}^{N_{s}} \sum_{s=1}^{N_{s}} A^{r s,-1} \Delta t \theta \frac{\partial \dot{\gamma}^{r}}{\partial \tau^{r}}\right|_{t}\left[\underset{\approx}{\boldsymbol{\Lambda}}: \operatorname{sym}\left(\underline{\boldsymbol{l}}^{r} \otimes \underline{\boldsymbol{n}}^{r}\right)\right] \otimes\left[\underset{\approx}{\boldsymbol{\Lambda}}: \operatorname{sym}\left(\underline{\boldsymbol{l}}^{s} \otimes \underline{\boldsymbol{n}}^{s}\right)\right]
$$

\subsection{Validation of the Finite Element implementation}

The numerical implementation scheme is validated by comparison with the results provided by Zset solution [35, 63]. The $\theta$-method used in Zset solution (Eq. 38), deduced from Eq. 33 is solved by a Newton-Raphson method.

$$
K\left|\frac{\Delta \gamma^{s}}{\Delta t}\right|^{\frac{1}{n}}-\left(\mid\left(\tau^{s, t+\theta \Delta t}-\chi^{s, t+\theta \Delta t} \mid-r^{s, t+\theta \Delta t}-\tau_{0}^{s}\right)=0\right.
$$

The validation procedure is performed on a single crystal stainless steel where the data are collected from [28]. It should be mentioned that the slip interaction matrix is formulated for a BCC structure $\left(h_{i}=1 ; \forall i=1, \ldots, 8\right)$. A $10 \mathrm{~mm}$ diameter and $20 \mathrm{~mm}$ length cylinder is considered. It is embedded on one side and undergone a tensile loading up to 4\%. 8-node linear brick elements are used for the meshing, more than 400 elements are generated with a full integration. The comparison between both approaches exhibits a good agreement (Fig. 9). 


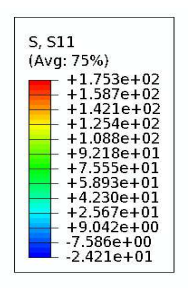

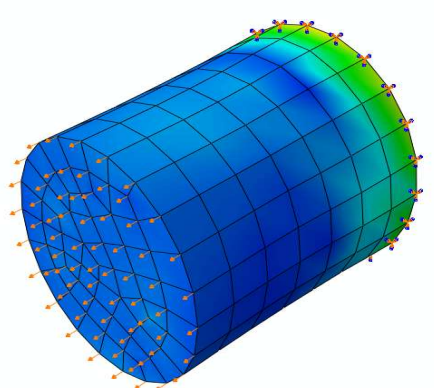

(a) UMAT

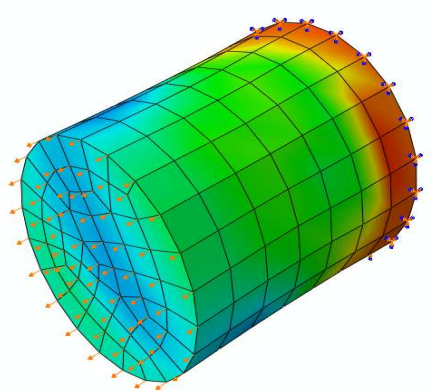

(c) UMAT
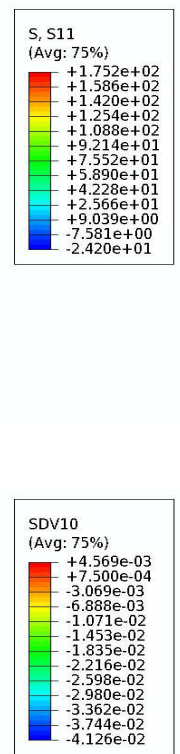

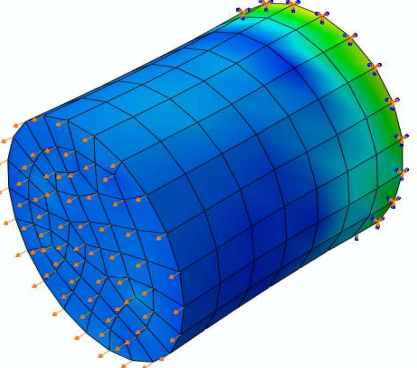

(b) ZMAT

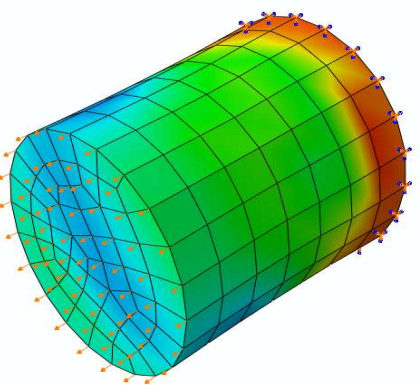

(d) ZMAT

Figure 9: Comparison of the stress field (a-b) in the loading direction and the shear stress (c-d) of the Schmid-Boas slip system G1 (see Tab. A.8) obtained by the integration method developed in this work (a-c) and those provided by Zset Solution [63] (b-d)

\subsection{Finite Element pre-processing}

Voronoï cell tesselations are used to generate the virtual microstructure of X38CrMoV51 martensitic steel. The computational methodology consists in performing ordinary Voronoï tesselations [72, 73] in a spatial domain (REV) of $150 \times 150 \times 50 \mu^{3}$. Tesselations generation is given by Neper software [40, 74]. 173 polycrystalline aggregates representing the potentials sites of the martensitic packets (First order Tesselation) are generated (Fig. 10a). Then, from this first order tesselation, starting from these sites, randomly oriented segments are generated where additional nucleation points are placed from which a second Voronoï tesselation is performed (second order Tesselation, Fig. 10b). 6 parallel laths are considered in each block except for one where only 2 laths are considered, achieving a total $N=1034(172 \times 6+2)$ Voronoï cells. The Crystallographic orientations of the laths are not explicitly considered in the virtual microstructure. In fact, the laths are grouped in a parallel manner in each block and are randomly oriented from one to another block. PAGs are constituted of 4 packets of laths except for one PAG including only 2 laths achieving 44 PAG.

Due to the relative motion between the surfaces of the tool and the part, a non isotropic microstructure (elongated PAG and laths) is generated close to the surface of the tool. For this purpose, the Voronoï cell centers related to the upper half of RVE are translated to represent elongated virtual microstructure (Fig 11a-11b). Furthermore, micro-hardness measurements reveal a hardening close to the free surface. This hardening is considered into the FE simulation by increasing the critical 


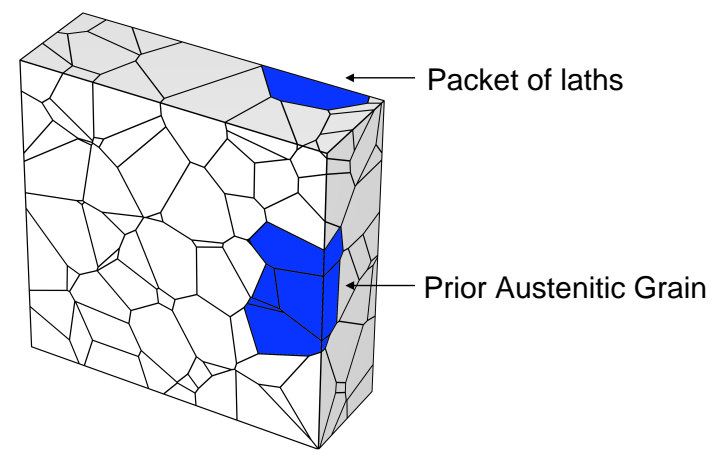

(a)

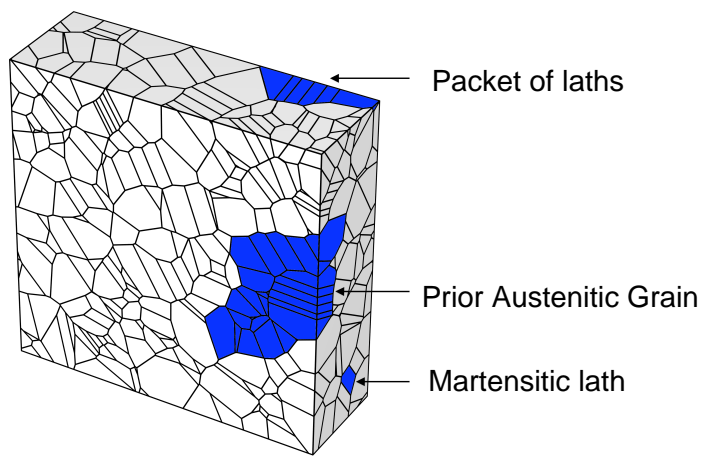

(b)

Figure 10: Virtual microstructure generation by Voronoï tesselations: first order tesselation (a); second order tesselation (b)

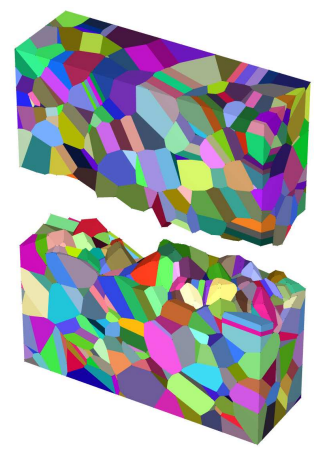

(a)

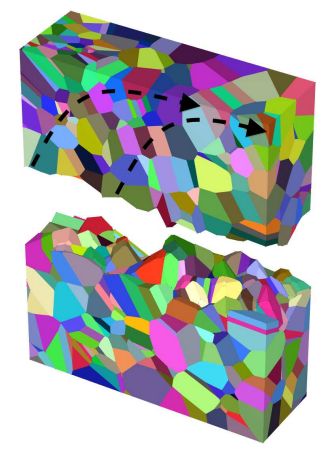

(b)

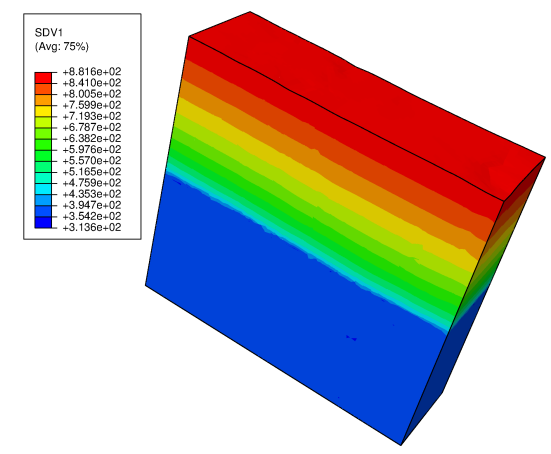

(c)

Figure 11: Isotropic lath morphology (a); Non isotropic morphology (b); Surface Hardening by introducing an increase of the resolved shear stress $\tau_{0}^{s}(\mathrm{c})$

Moreover, the martensitic phase transformation requires particular crystallographic relationship between the laths (BCC) and the matrix (Face-centered Cubic: FCC). In this study, the Kurdjumov-Sachs (KS) relationships are assumed since the $\mathrm{X} 38 \mathrm{CrMoV5}-1$ microstructure is similar to a low carbon steel [75, 76]. Considering the $\gamma / \alpha$ crystallographic planes relationship $\{111\}_{\gamma} \|\{110\}_{\alpha^{\prime}}$ and crystallographic orientation relationships $<110>_{\gamma} \|<111>_{\alpha^{\prime}}, 24$ variants are identified (see table A.9 in appendix). These variants are assigned to each PAG containing 4 packets where each packet contains 6 laths sharing the same habit. The 24 variants are randomly distributed (Fig. 12b). In such manner, global martensitic laths orientations distribution is isotropic (Fig. [12C). For the layers close to the surface of the $\mathrm{REV}$, the crystallographic texture given by KS relationships can be replaced by an experimental texture provided by EBSD measurements for a better consideration of the experimental evidence. The last effect to be considered deals with the surface roughness of the REV. Based on experimental results, the ultimate layer of RVE is differently meshed by a node coordinate displacement. 


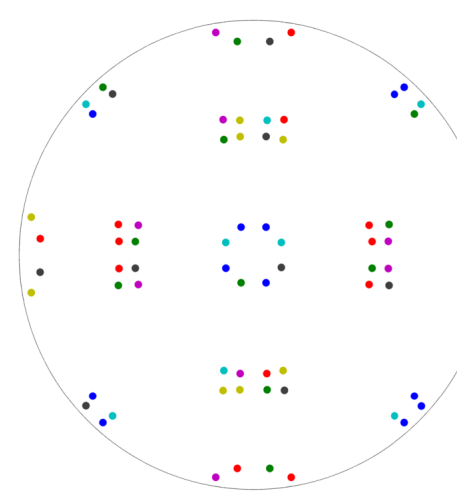

(a) KS orientation relationship

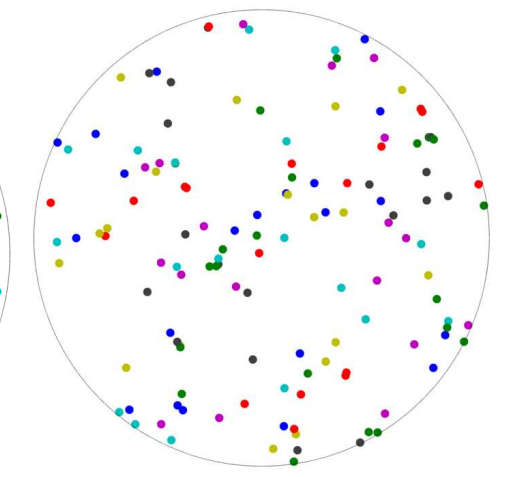

(b) Orientation of the austenitic (c) structure

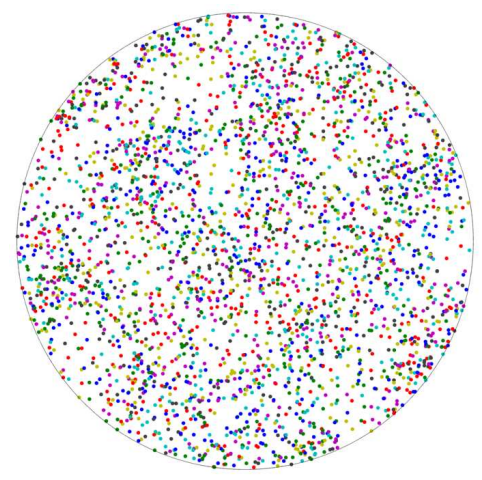

(c) Orientation of the martensitic structure

Figure 12: (100) pole figures (a) KS orientations relationship, (b) PAG, (c) martensitic lath

421

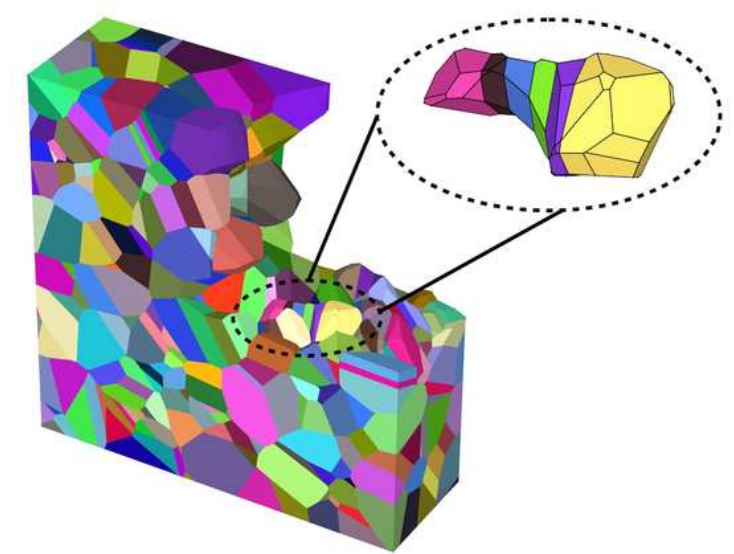
the mean size of the finite elements.

$$
l_{c}=0.5 r_{c} V_{k}^{\frac{1}{3}} ; \quad r_{c}=1.15
$$

where $V_{k}$ is the volume of the Voronoï cell $k$ and $r_{c}$ a fixed parameter related to

The FE meshing of Voronoï cells is not trivial. The meshing technique proposed by Quey [40] and implemented on Neper software is adopted. It includes an accurate discretization for taking into account the martensitic laths. This procedure needs some modifications in the morphology of the generated virtual microstructure. The edges of the Voronoï polyhedra with a short length $\left(l \leq l_{c}\right.$, see Eq. [39) were eliminated. Therefore, some faces and vertexes of the Voronoï cells vanishes and the virtual microstructure is rebuilt by interpolation techniques as illustrated in Fig. 13.

Figure 13: Improvement of the Voronoï cell shape for better mesh criteria

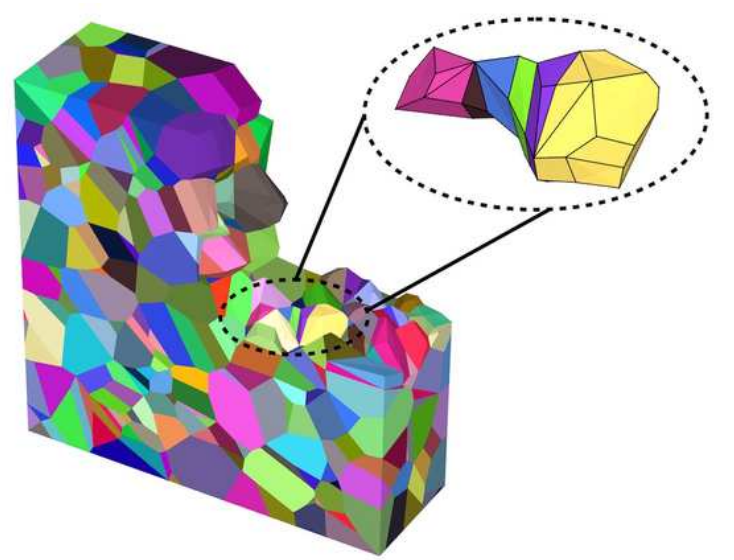

Then, a free meshing technique using quadratic tetrahedral elements with a full integration (namely C3D10 in ABAQUS software) is performed. 84177 elements are generated corresponding to an average value of 82 elements per cell which is relevant 
for a multi-scale analysis [40]. The meshing analysis shows that the aspect ratio for only less than $0.59 \%$ of the finite elements is inadequate. The distribution of the elements per lath (e.g. Voronoï cell) is illustrated in Fig. 14a, The meshing and the boundary conditions prescribed on the RVE are shown in Fig. 14b, Two types of loading are considered, a tensile loading with a maximal strain of $\varepsilon=8 \%$ and a cyclic loading with $R_{\varepsilon}=\frac{\varepsilon_{\min }}{\varepsilon_{\max }}=0.1$.

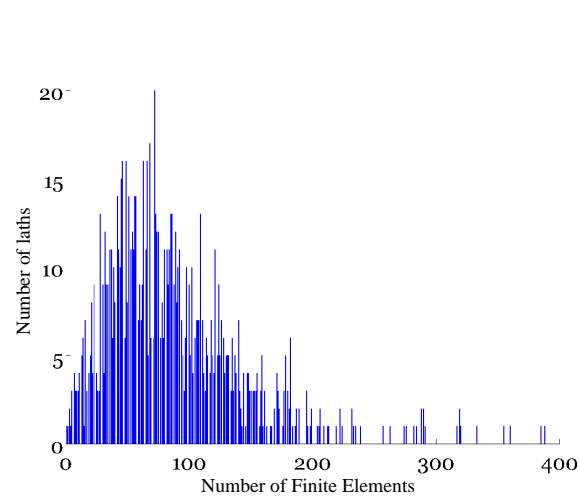

(a)

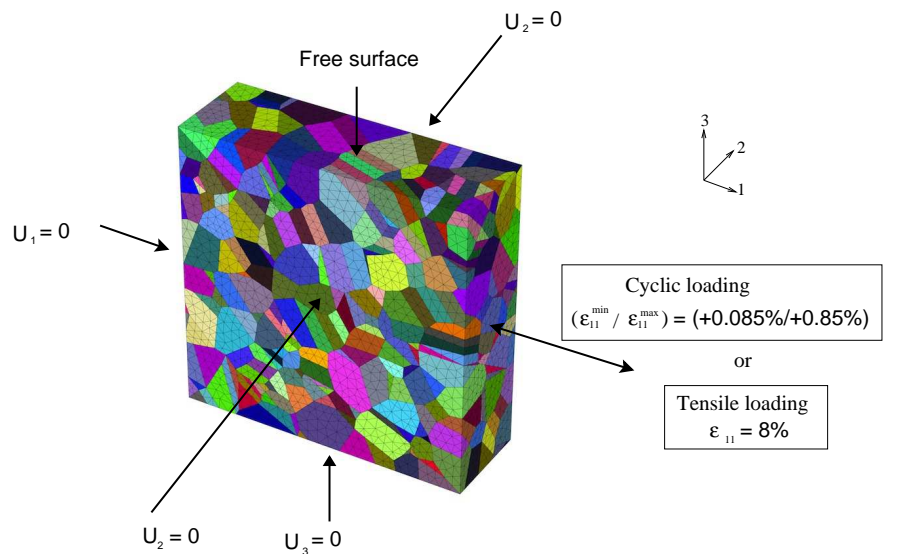

(b)

Figure 14: Distribution of the number of elements per lath (a); Meshing of the RVE and prescribed boundary conditions (b)

A FE parallel calculation is undertaken in ABAQUS software where the FETI (Finite Element Tearing and Interconnecting) method is used [77]. It allows the partition of the spatial domain into a set of disconnected sub-domains, each being assigned to an individual processor. In the present study, 8 processors with a clock rate of $2.8 \mathrm{GHz}$ and a Random Access Memory of $30 \mathrm{~GB}$ per node are considered for each Finite Element calculation.

\section{Results and discussions}

\subsection{Present and future issues of the approach}

The knowledge of the actual mechanical loadings and the subsurface behavior of bodies in relative movement (sliding) requires the development of appropriate constitutive laws. In most cases, isotropic and macroscopic behavior is assumed. However, detailed investigations and microscopic observations on damage mechanisms reveal that the number of grains experiencing and bearing the shear strains at the extreme surfaces is limited [43, 54]. Therefore it becomes mandatory to distinguish the mechanical behavior at the subsurface from the bulk. The grain boundaries emerging at surface, the interaction between surface and active dislocations in these grains, rupturing of well-orientated secondary phases (carbides, non spherical precipitates), rolling of spherical second phases or debris and specific crystallographic orientation of each grain cause various damage mechanisms. These mechanisms can simultaneously be activated and even coupled, accelerating the processes and accumulation of damages. Under incremental shear straining the subsurface plastic yielding can 
occur. The magnitude of the plastic yielding decreases inwards from the surface. One can observe that in addition to texturing of the martensitic laths and subsequent PAGs beneath the surface of pins, micro-cavities (with different sizes) are also formed under excessive and cumulative shear straining (See Fig. 8 in [54]). At triple grains junction, the orientation of each laths packet and different crystallographic orientation of grains lead to micro-tearing and to the formation of micro-cavities as a consequence of the shear strain accumulations and an important stress/strain tri-axiality state. The micro-tearing located beneath the working surface can generate wear debris. Under such complex conditions the macroscopic and isotropic constitutive laws might fail to well describe the subsurface behavior. Defining a damage process zone beneath the working surface of pins used in tribology investigations, Boher et al have proposed a wear model based on cumulative shear straining [78]. The fundamental of this model is inspired from Manson-Coffin law for LCF life prediction. The authors suppose that debris are emitted once the cumulative shear strain increment reaches the shear ductility. In this model, the plastic shear strain increment is evaluated by a macroscopic finite element simulation of a pin on a rotating disc. At this scale of investigation, the microstructure features drastically influence the behavior of the subsurface and cannot be neglected. Our approach can definitively improve the assessment of the cumulative shear strain increment required in the wear model [78].

The model results are reported hereafter under tensile and cyclic loading conditions for emphasizing the local behavior of individual lath, lath packets and PAGs. Many numerical results are extracted from the FE analysis for evaluating the stress and strain components at different scales such as:

- von Mises stress and inelastic strains,

- number of active slip systems,

- accumulated intrinsic dissipation $\Theta$, i.e. the integral in time of Eq. 15,

Some of these results are compared with experimental tensile tests using DIC analysis.

\subsection{Tensile loading}

Several assumptions concerning the orientations relationships (KS or experimental), the shape of the martensitic laths (anisotropic aspect), the shape of the surface (flat or rough) are examined (table 6).

Table 6: Tensile test conditions used for numerical simulations

\begin{tabular}{cccc}
\hline Test condition & Crystallographic Orientations & Surface hardening & shape of the surface \\
\hline 1 & KS & non & flat \\
2 & KS & yes & flat \\
3 & experimental & yes & flat \\
4 & experimental & yes & rough \\
\hline
\end{tabular}


Fig. 15 illustrates the results (i.e. maps of the von Mises stress (a); the number of active slip systems (b); the intrinsic dissipation (c)) for a total deformation of $8 \%$ and test condition 1 . The von Mises stress shows significant stress concentrations close to the martensitic laths or PAG boundaries which are mainly influenced by the crystallographic orientations. The number of active slip systems does not show specific stress localization. The intrinsic dissipation reveals the zones where the inelastic strain is high. Unlike the macroscopic modeling of the behavior where the free surface of the specimen remains unchanged, one can notice that multi-scale crystal plasticity well predicts the free surface roughening.

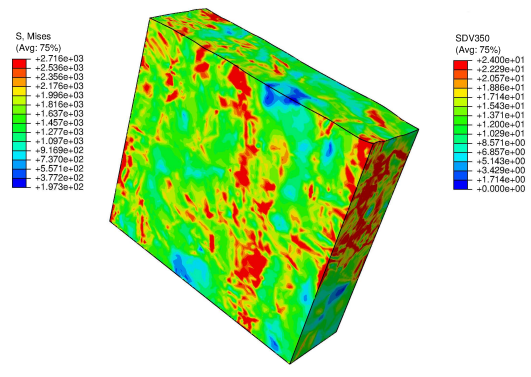

(a)

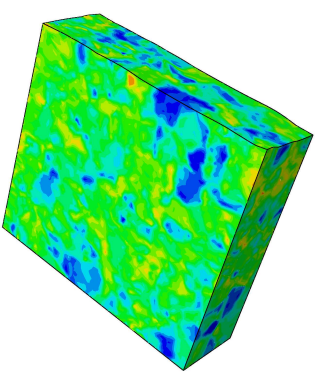

(b)
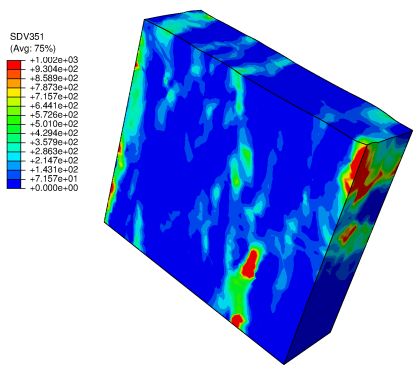

(c)

Figure 15: Equivalent von Mises stress component (a), number of active slip systems (b) and intrinsic dissipation (c) for the test conditions 1

Test conditions 2, 3 and 4 (table 6) are examined in order to be more realistic. Fig. 16a illustrates that the macroscopic strain-stress responses at the scale of the RVE are very close whatever test conditions. However, important variation in the local von Mises stress are found after $8 \%$ straining. The von Mises stress maps reveal that the stress concentrations are greatly influenced by the initial orientation relationships (Fig. 16b-16c). This effect is predominant whithin the upper half of the RVE where the crystallographic orientation relationships are identified by EBSD. Nevertheless, it should be mentioned that the lower half of the RVE is also affected by stress concentration while KS orientation relationships are identical for all simulated test conditions. When the rough surface is modeled (test condition 4 ), the stress fields drastically change at the free surface while no such variation is observed for the other test conditions (2 and 3).

The stress-strain curves are numerically simulated for test condition 4 at different scales (scale of the RVE (a); of the PAG (b); of the lath packets (d); of the laths (d), Fig. 17) . First, one can notice that the stress-strain response obtained at the RVE scale is quite similar to the tensile experimental curve and also to the simulated stress-strain curve using the mean field scale transition rule (Fig. $8 \mathrm{~d}$ ). It means that the parameters identified using the $\beta$ model and the FE method are consistent. The strain field heterogeneity increases when the scale decreases. Indeed, at the martensitic lath scale, a maximal strain level of 0.9 can be reached. The maximal stress can be $250 \%$ higher than the global macroscopic stresses (measured or simulated). The number of the active slip systems strongly depends on the crystallographic orientation of a given lath and its morphology, this is why, when 


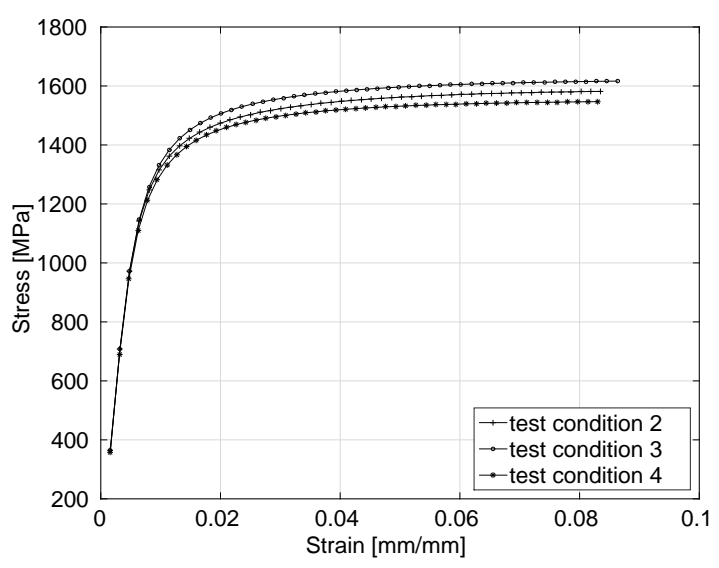

(a)

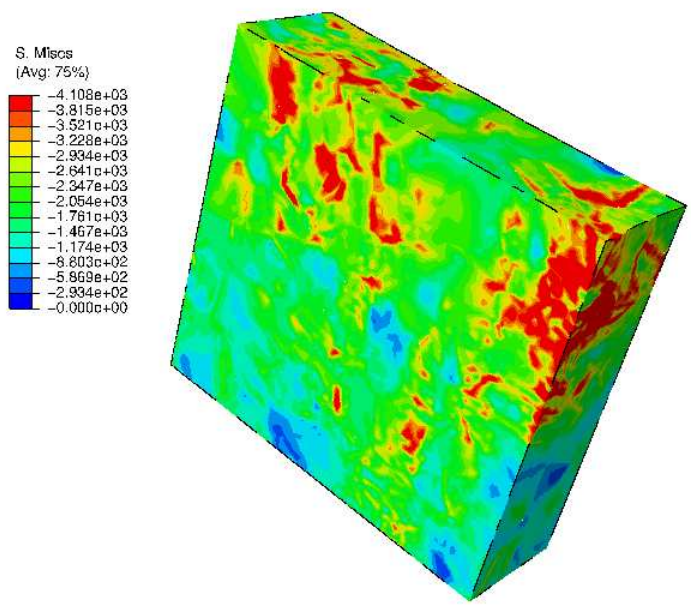

(c)
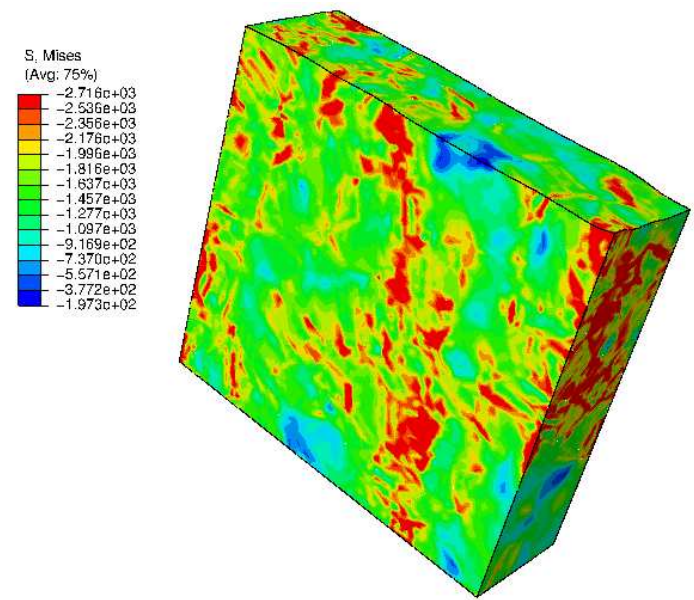

(b)
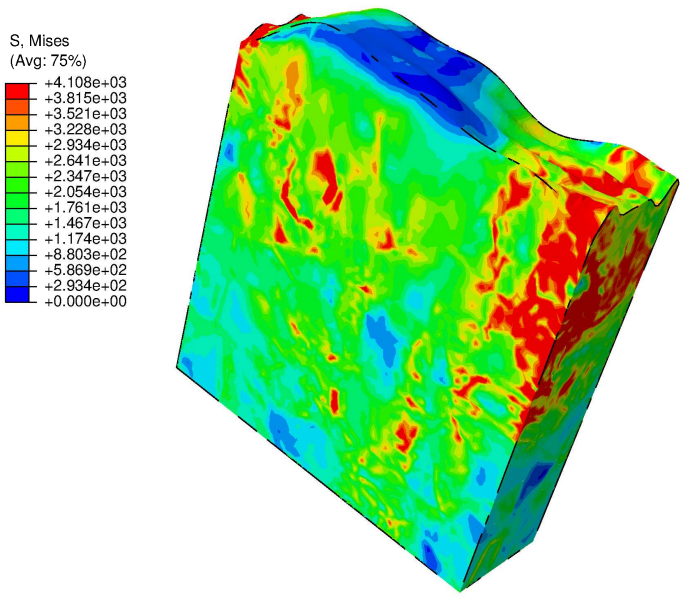

(d)

Figure 16: Macroscopic Strain-Stress response for test conditions 2, 3 and 4 (a); von Mises stress field for the test conditions 2 (b); 3 (c) and 4 (d)

the simulated stresses for two laths may be very close, their local strains could be very different. The comparison between the strain fields calculated by FE and measured by DIC is not trivial. It requires a high-performance digital image correlation technique allowing a mapping of the strain fields at a microscopic scale [46]. In fact, there is shortcoming to apply 2D strain field measurements on microstructure with $3 \mathrm{D}$ distribution of phases and precipitates. Also, the number of data collected (e.g. strain field) from DIC measurements (2D) and RVE simulations (3D) are very different. Therefore, the number of data is collected for each major strain level. Then the maximum value for the data collected, is extracted. At the end, a ratio of each data collected over this maximum value is calculated. Through the normalization method, experimental and simulated results can be compared as a function of major strain levels (Fig. 18, for the test conditions 4 and a macroscopic major strain of 3\%). 

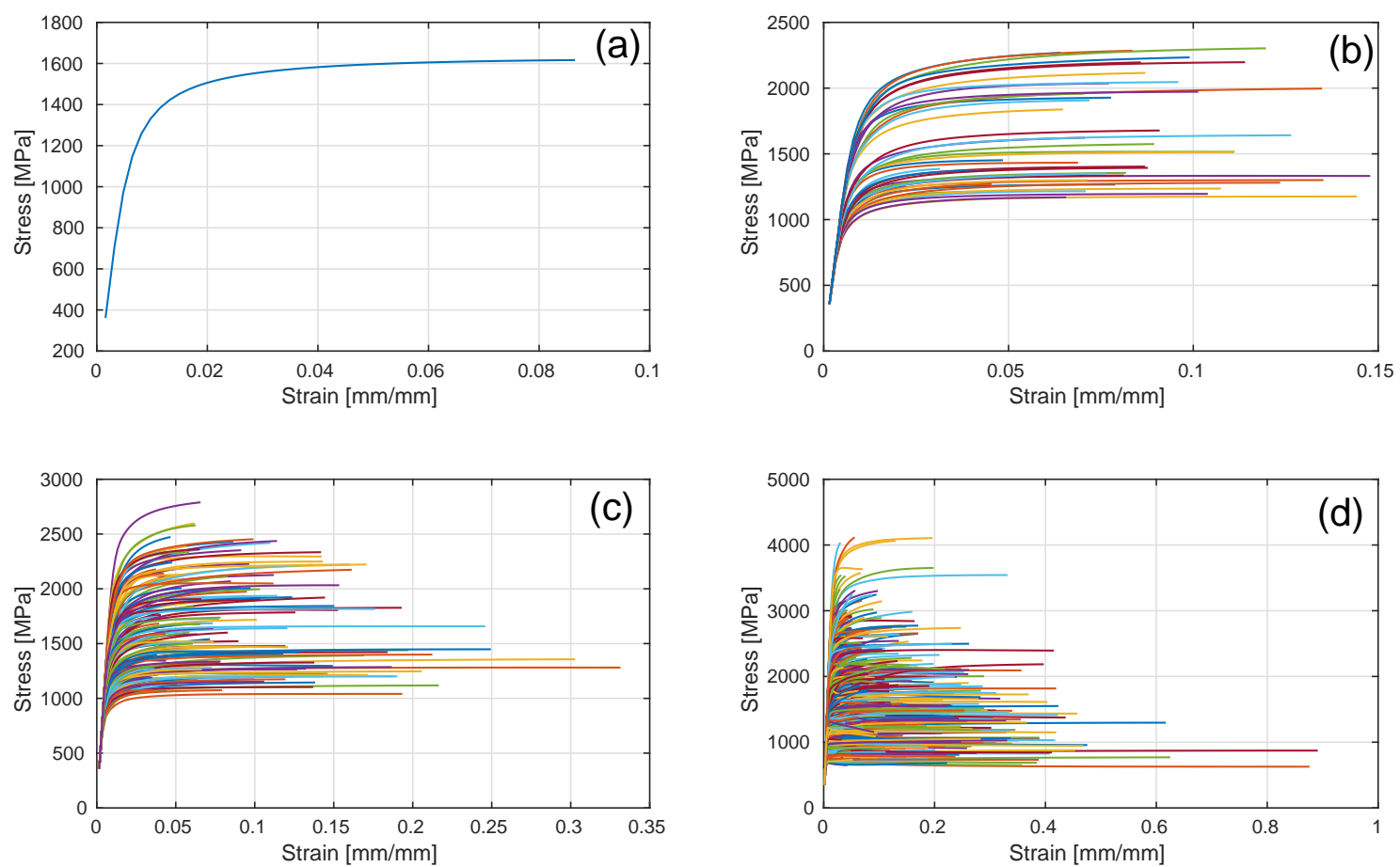

Figure 17: Strain-Stress curves under a tensile loading at different scales: (a) macroscopic, i.e. RVE behavior (well reproducing the experimental variation of the stress versus the strain in a tensile condition) ; (b) different PAG; (c) different lath packets; and (d) different martensitic laths (showing a huge scattering) using test conditions 4 (experimental crystallographic orientation provided by EBSD at the upper half of the RVE, considering a surface hardening through a decrease of the resolved shear stress and taking into account a roughness of the RVE surface). One can notice that the scattering in (b) and (c) are reduced in comparison with (d) due to the scale of the evaluations and tending towards the macroscopic curve (a).

\subsection{Cyclic loading}

9 cyclic tests (see Fig. 14b) are conducted on the RVE for different conditions (table 7). Similarly to the tensile tests, different assumptions can be made. A flat surface is considered. In one case, a microstructure is randomly generated by using the Kurdjumov and Sachs (KS) relationships with (test condition 6) or without (test condition 5) considering hardening of the surface on the upper half of the RVE as illustrated in Fig. 11c, In the second case, the measured crystallographic relationships by EBSD is used to generate this microstructure (test condition 7).

Table 7: Test conditions investigated in the numerical simulations for the cyclic tests

\begin{tabular}{cccc}
\hline Test condition & Crystallographic Orientations & Surface hardening & shape of the surface \\
\hline 5 & KS & non & flat \\
6 & KS & yes & flat \\
7 & experimental & yes & flat \\
\hline
\end{tabular}

Fig. 19a compares the average value of the stress-strain loops obtained by FE simulation at the RVE scale for the test conditions 5 to 7 and the cycles 1 and 9 . This average value is in fact given by the extracted computed stress-strain values 


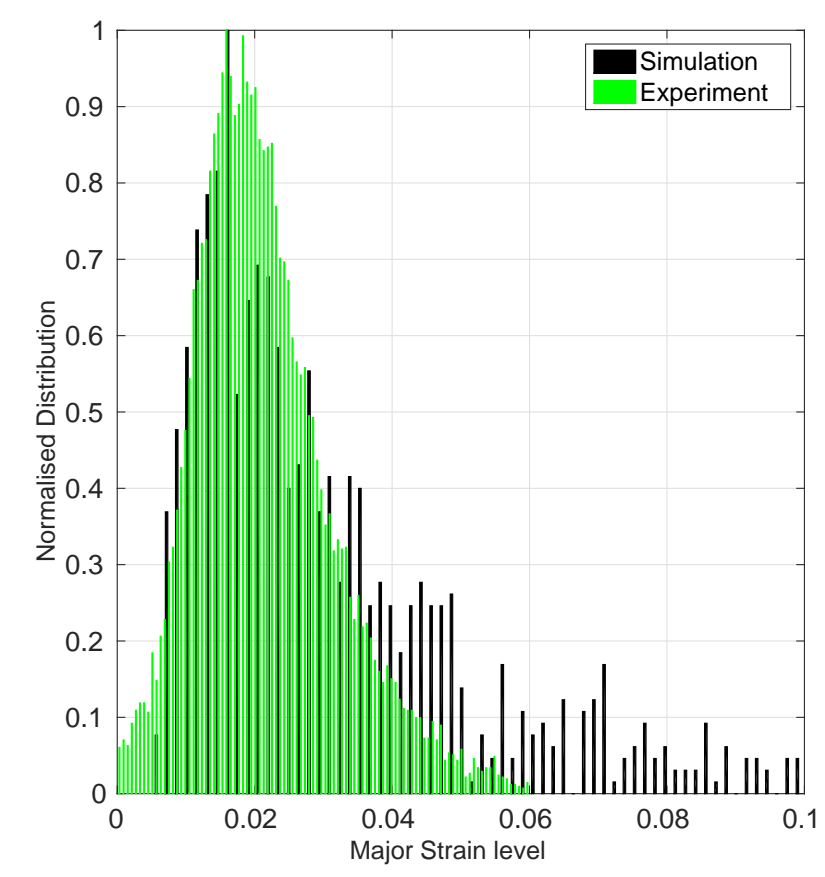

Figure 18: Distribution of the laths over the strain levels for an average strain level of $3 \%$

at the level of laths, packets of laths and PAG. The trend for the test conditions 6 and 7 are close eventhough the considered textures are different (KS relations and experimental by EBSD) whereas a significant change is observed in the stress level of test condition 5 for which no surface hardening is considered. Fig. 19b-19d gives the local von Mises stress at the maximal strain level of the cycle 9 for each test condition. For the test conditions 5 and 6 , the surface hardening introduced at the upper half of the RVE influences the stress distribution at the lower half even if the same crystallographic relations are considered. Moreover, a similar surface hardening is considered but with different crystallographic relations (test conditions 6 and 7 ), it is observed that the stress distribution is quite different inside the RVE while the RVE strain-stress response are similar (Fig. 19a). Fig. 20 shows the strain-stress responses for test condition 7 at different scales and for cycles 1 and 9 . At the RVE scale, a plastic shakedown is observed between cycle 1 and 9 (Fig. 20 a-d). At the PAG scale, the plastic shakedown is not yet completed (Fig. 20 b-e). In fact, at the laths scale, the plastic ratcheting is very active, thus influencing the behavior at the PAG level. Fig. 20 c-f show clearly the enlarging of the stress-strain hysteresis loop.

\section{Conclusions}

The surface modeling of tool steels are investigated at room temperature by experimental testing and numerical simulation. The elasto-viscoplastic equations are formulated at the scale of the slip systems considering an isotropic and kinematic hardening variables to predict both tensile and cyclic loadings. Phenomenological constitutive equations of Meric and Cailletaud [34, 35] are adapted for the 

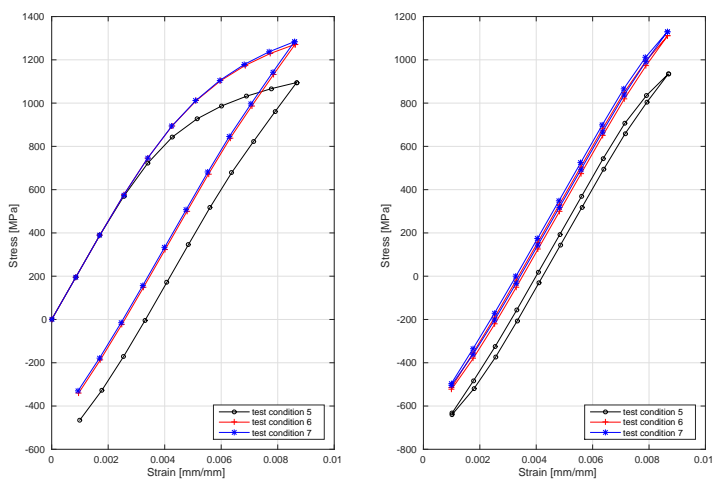

(a)

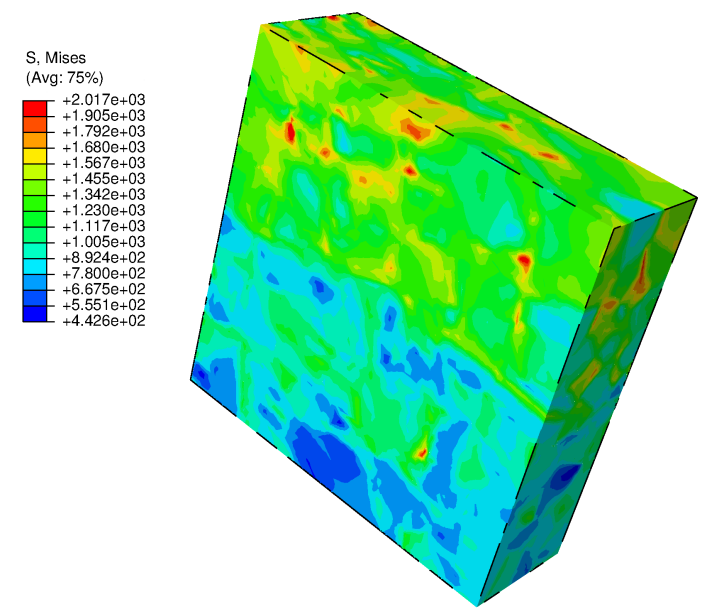

(c)
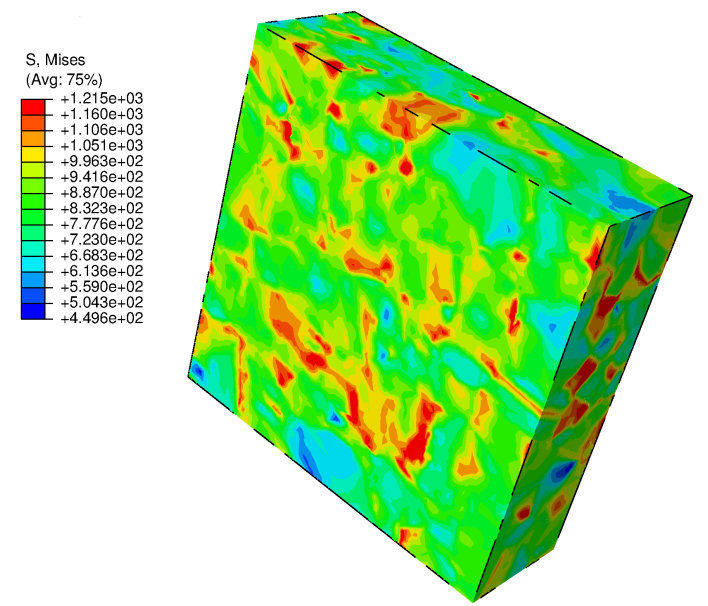

(b)
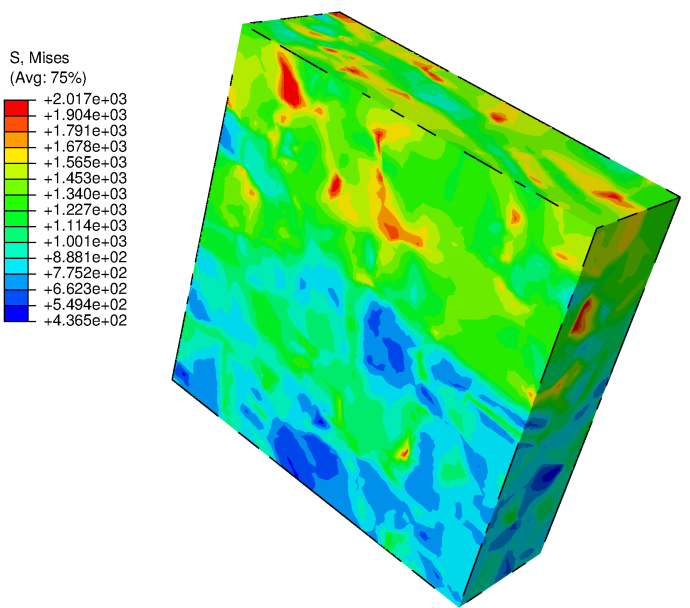

(d)

Figure 19: Macroscopic Strain-Stress response for test conditions 5, 6 and 7 for the $1^{\text {st }}$ (a) and the $9^{\text {th }}$ (b) cycle; von Mises stress field for the test conditions 5 (b); 6 (c) and 7 (d) at the maximal strain of the $9^{\text {th }}$ cycle

X38CrMoV5-1 double tempered martensitic steel with randomly oriented laths. The thermodynamic framework of the non reversible phenomena is respected at the levels of the slip systems, laths, packets of laths and PAG.

The following points can be addressed:

- DIC technique is used to propose a relevant RVE for this steel and also to determine the local strain fields.

- Two mean field approaches [18, 19] are applied for identifying the parameters of the constitutive laws. These methods ensure the transition between the slip systems and the RVE behavior.

- A full field approach using FE method takes into account explicitly the microstructure features of the steel (martensitic laths, crystallographic orientations, grain morphology). Voronoï tesselation is used to generate the virtual 

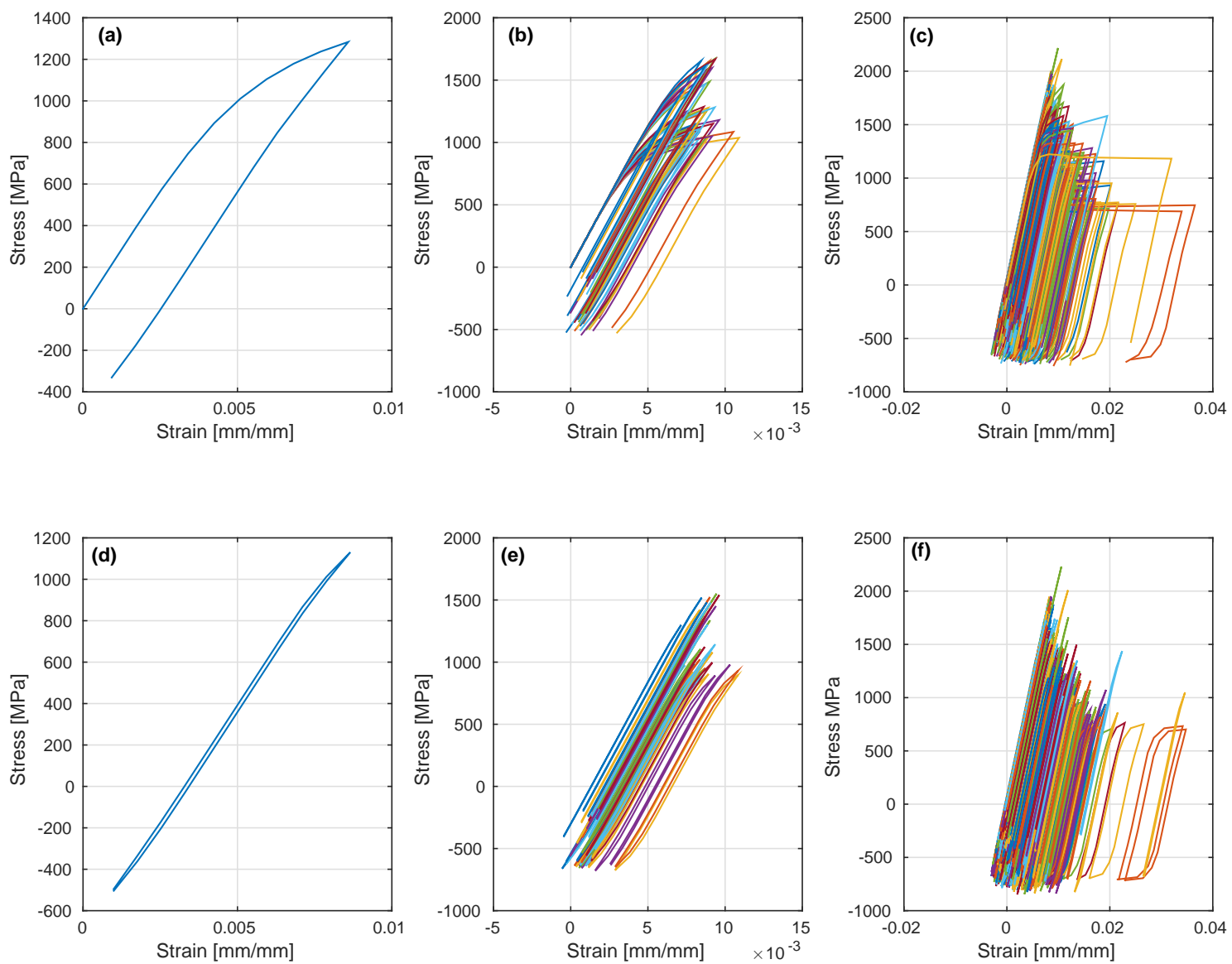

Figure 20: Strain-Stress curves under cyclic loading at the scale of the RVE (a,d), the PAG (b,e) and the martensitic laths $(c, f)$ at the maximal strain of the $1^{\text {st }}(\mathrm{a}, \mathrm{b}, \mathrm{c})$ and the $9^{\text {th }}$ cycle $(\mathrm{d}, \mathrm{e}, \mathrm{f})$ for the test conditions 7

microstructure [40]. This approach is based on a direct resolution scheme developed by Peirce [15, 16] and a predictor/corrector alogrithm of Simo [70].

- The virtual microstructure is generated either based on EBSD measurements or Kurdjumov-Sachs crystallographic relationships between the matrix (FCC) and the laths $(\mathrm{BCC})$. It is observed that the surface hardening of the layer of RVE influences the other half of the RVE behavior for different crystallographic orientations. The effect of the surface aspects (plane or rough) is also investigated.

- The reliability of modeling approach is assessed for different loading conditions and microstructural aspects. An acceptable agreement between DIC measurements and the FE simulation is found for the local distribution of the strain fields (only under tensile loading condition).

- Cyclic loading simulations show that a plastic shakedown occurs at RVE level. At the PAG scale, the plastic shakedown is not totally achieved. At the laths scale, plastic ratcheting is very active. Enlargement of stress-strain loops are clearly observed. This enlargement is less at the level of the PAG. 


\section{Acknowledgement}

The authors would like to gratefully acknowledge the scientific grouping CALMIP for its support with regard to the FE calculations. Most of them were run on a supercomputer which was made available to the researchers. The authors deeply acknowledge Dr. Christine Boher from Mines-Albi \& Institut Clément Ader, for many valuable and fruitful discussions and exchanges on the wear subsurface behavior of bodies in movement.

\section{Appendix A.}

Appendix A.1. Interaction Matrix and Schmid-Boas and Miller notation systems

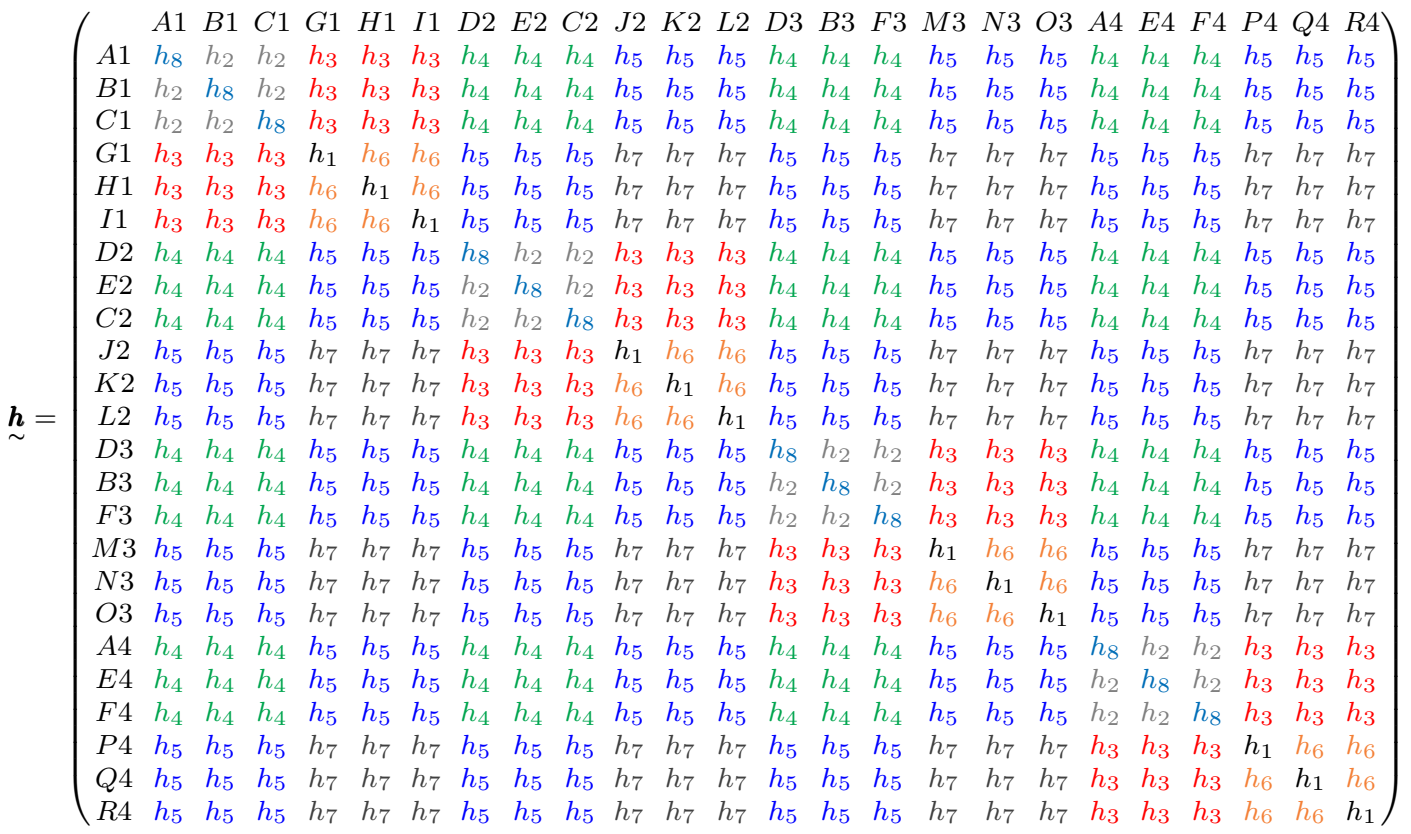

Table A.8: Normalized Schmid-Boas and Miller notation systems

\begin{tabular}{|c|c|c|c|c|c|c|c|c|c|c|c|c|}
\hline Schmid-Boas & A1 & B1 & $\mathrm{C} 1$ & G1 & H1 & I1 & D2 & E2 & $\mathrm{C} 2$ & $\mathrm{~J} 2$ & $\mathrm{~K} 2$ & L2 \\
\hline Slip plane $\underline{\boldsymbol{l}}^{s}$ & $\frac{(110)}{\sqrt{2}}$ & $\frac{(10 \overline{1})}{\sqrt{2}}$ & $\frac{(01 \overline{1})}{\sqrt{2}}$ & $\frac{(11 \overline{2})}{\sqrt{6}}$ & $\frac{(1 \overline{2} 1)}{\sqrt{6}}$ & $\frac{(\overline{2} 11)}{\sqrt{6}}$ & $\frac{(110)}{\sqrt{2}}$ & $\frac{(101)}{\sqrt{2}}$ & $\frac{(01 \overline{1})}{\sqrt{2}}$ & $\frac{(1 \overline{1} 2)}{\sqrt{6}}$ & $\frac{(12 \overline{1})}{\sqrt{6}}$ & $\frac{(211)}{\sqrt{6}}$ \\
\hline $\begin{array}{l}\text { Slip direction } \\
\underline{\boldsymbol{n}}^{s}\end{array}$ & $\frac{[111]}{\sqrt{3}}$ & $\frac{[111]}{\sqrt{3}}$ & $\frac{[111]}{\sqrt{3}}$ & $\frac{[111]}{\sqrt{3}}$ & $\frac{[111]}{\sqrt{3}}$ & $\frac{[111]}{\sqrt{3}}$ & $\frac{[\overline{1} 11]}{\sqrt{3}}$ & $\frac{[\overline{1} 11]}{\sqrt{3}}$ & $\frac{[\overline{1} 11]}{\sqrt{3}}$ & $\frac{[\overline{1} 11]}{\sqrt{3}}$ & $\frac{[\overline{1} 11]}{\sqrt{3}}$ & $\frac{[\overline{1} 11]}{\sqrt{3}}$ \\
\hline Schmid-Boas & D3 & B3 & F3 & M3 & N3 & O3 & $\mathrm{A} 4$ & $\mathrm{E} 4$ & $\mathrm{~F} 4$ & $\mathrm{P} 4$ & $\mathrm{Q} 4$ & $\mathrm{R} 4$ \\
\hline Slip plane $\underline{\boldsymbol{l}}^{s}$ & $\frac{(110)}{\sqrt{2}}$ & $\frac{(10 \overline{1})}{\sqrt{2}}$ & $\frac{(011)}{\sqrt{2}}$ & $\frac{(1 \overline{1} \overline{2})}{\sqrt{6}}$ & $\frac{(112)}{\sqrt{6}}$ & $\frac{(\overline{2} \overline{1} 1)}{\sqrt{6}}$ & $\frac{(1 \overline{1} 0)}{\sqrt{2}}$ & $\frac{(101)}{\sqrt{2}}$ & $\frac{(011)}{\sqrt{2}}$ & $\frac{(\overline{1} \overline{1} \overline{2})}{\sqrt{6}}$ & $\frac{(\overline{1} 21)}{\sqrt{6}}$ & $\frac{(2 \overline{1} 1)}{\sqrt{6}}$ \\
\hline $\begin{array}{l}\text { Slip direction } \\
\underline{\boldsymbol{n}}^{s}\end{array}$ & $\frac{[1 \overline{1} 1]}{\sqrt{3}}$ & $\frac{[1 \overline{1} 1]}{\sqrt{3}}$ & $\frac{[1 \overline{1} 1]}{\sqrt{3}}$ & $\frac{[1 \overline{1} 1]}{\sqrt{3}}$ & $\frac{[1 \overline{1} 1]}{\sqrt{3}}$ & $\frac{[1 \overline{1} 1]}{\sqrt{3}}$ & $\frac{[11 \overline{1}]}{\sqrt{3}}$ & $\frac{[11 \overline{1}]}{\sqrt{3}}$ & $\frac{[11 \overline{1}]}{\sqrt{3}}$ & $\frac{[11 \overline{1}]}{\sqrt{3}}$ & $\frac{[11 \overline{1}]}{\sqrt{3}}$ & $\frac{[11 \overline{1}]}{\sqrt{3}}$ \\
\hline
\end{tabular}


Appendix A.2. Variant orientation of a PAG according to the KS relationship

In the present investigation, 24 laths and 4 blocks (6 laths per block) are included in each PAG. The KS relationship assumes that a block is defined by a parallelism relationship between two crystallographic planes given by the $\alpha^{\prime}$ (martensitic lath) and $\gamma$ (austenitic grain) phases. The parallelism relationship for the variant orientations between two crystallographic directions is recalled in Table A.9.

Table A.9: Variant orientation of a PAG according to the KS relationship

\begin{tabular}{|c|c|c|c|}
\hline Block & Parallelism & Variant & Parallelism \\
\hline \multirow[t]{6}{*}{1} & $\left(\begin{array}{lll}1 & 1 & 1\end{array}\right)_{\gamma} \|\left(\begin{array}{lll}0 & 1 & 1\end{array}\right)_{\alpha^{\prime}}$ & 1 & {$\left[\begin{array}{lll}\overline{1} & 0 & 1\end{array}\right]_{\gamma} \|\left[\begin{array}{lll}\overline{1} & \overline{1} & 1\end{array}\right]_{\alpha^{\prime}}$} \\
\hline & & 2 & {$\left[\begin{array}{lll}\overline{1} & 0 & 1\end{array}\right]_{\gamma} \|\left[\begin{array}{lll}\overline{1} & 1 & \overline{1}\end{array}\right]_{\alpha^{\prime}}$} \\
\hline & & 3 & {$[0 \overline{1} 1]_{\gamma} \|\left[\begin{array}{lll}\overline{1} & \overline{1} & 1\end{array}\right]_{\alpha^{\prime}}$} \\
\hline & & 4 & {$\left[\begin{array}{lll}0 & \overline{1} & 1\end{array}\right]_{\gamma} \|\left[\begin{array}{lll}\overline{1} & 1 & \overline{1}\end{array}\right]_{\alpha^{\prime}}$} \\
\hline & & 5 & 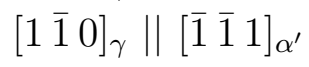 \\
\hline & & 6 & 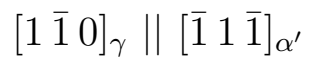 \\
\hline \multirow[t]{6}{*}{2} & $\left(\begin{array}{lll}1 & 1 & 1\end{array}\right)_{\gamma} \|\left(\begin{array}{lll}0 & 1 & 1\end{array}\right)_{\alpha^{\prime}}$ & 7 & {$\left[\begin{array}{lll}1 & 0 & \overline{1}\end{array}\right]_{\gamma} \|\left[\begin{array}{lll}\overline{1} & \overline{1} & 1\end{array}\right]_{\alpha^{\prime}}$} \\
\hline & & 8 & {$\left[\begin{array}{lll}1 & 0 & \overline{1}\end{array}\right]_{\gamma} \|\left[\begin{array}{lll}\overline{1} & 1 & \overline{1}\end{array}\right]_{\alpha^{\prime}}$} \\
\hline & & 9 & {$\left[\begin{array}{lll}\overline{1} & 1 & 0\end{array}\right]_{\gamma} \|\left[\begin{array}{lll}\overline{1} & 1 & 1\end{array}\right]_{\alpha^{\prime}}$} \\
\hline & & 10 & {$\left[\begin{array}{lll}\overline{1} & \overline{1} & 0\end{array}\right]_{\gamma} \|\left[\begin{array}{lll}\overline{1} & 1 & 1\end{array}\right]_{\alpha^{\prime}}$} \\
\hline & & 11 & {$\left[\begin{array}{lll}0 & 1 & 1\end{array}\right]_{\gamma} \|\left[\begin{array}{lll}\overline{1} & \overline{1} & 1\end{array}\right]_{\alpha^{\prime}}$} \\
\hline & & 12 & {$\left[\begin{array}{lll}0 & 1 & 1\end{array}\right]_{\gamma} \|\left[\begin{array}{lll}\overline{1} & 1 & \overline{1}\end{array}\right]_{\alpha^{\prime}}$} \\
\hline \multirow[t]{6}{*}{3} & $(\overline{1} 11)_{\gamma} \|\left(\begin{array}{lll}0 & 1 & 1\end{array}\right)_{\alpha^{\prime}}$ & 13 & 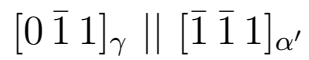 \\
\hline & & 14 & {$\left[\begin{array}{lll}0 & \overline{1} & 1\end{array}\right]_{\gamma} \|\left[\begin{array}{lll}\overline{1} & 1 & \overline{1}\end{array}\right]_{\alpha^{\prime}}$} \\
\hline & & 15 & {$\left[\begin{array}{lll}\overline{1} & 0 & \overline{1}\end{array}\right]_{\gamma} \|\left[\begin{array}{lll}\overline{1} & \overline{1} & 1\end{array}\right]_{\alpha^{\prime}}$} \\
\hline & & 16 & {$\left[\begin{array}{lll}\overline{1} & 0 & \overline{1}\end{array}\right]_{\gamma} \|\left[\begin{array}{lll}\overline{1} & 1 & \overline{1}\end{array}\right]_{\alpha^{\prime}}$} \\
\hline & & 17 & {$\left[\begin{array}{lll}1 & 1 & 0\end{array}\right]_{\gamma} \|\left[\begin{array}{lll}\overline{1} & \overline{1} & 1\end{array}\right]_{\alpha^{\prime}}$} \\
\hline & & 18 & {$\left[\begin{array}{lll}1 & 1 & 0\end{array}\right]_{\gamma} \|\left[\begin{array}{lll}\overline{1} & 1 & \overline{1}\end{array}\right]_{\alpha^{\prime}}$} \\
\hline \multirow[t]{6}{*}{4} & 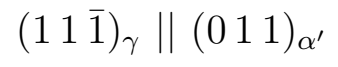 & 19 & {$\left[\begin{array}{lll}\overline{1} & 1 & 0\end{array}\right]_{\gamma} \|\left[\begin{array}{lll}\overline{1} & \overline{1} & 1\end{array}\right]_{\alpha^{\prime}}$} \\
\hline & & 20 & {$\left[\begin{array}{lll}\overline{1} & 1 & 0\end{array}\right]_{\gamma} \|\left[\begin{array}{lll}\overline{1} & 1 & \overline{1}\end{array}\right]_{\alpha^{\prime}}$} \\
\hline & & 21 & {$[0 \overline{1} \overline{1}]_{\gamma} \|\left[\begin{array}{lll}\overline{1} & 1 & 1\end{array}\right]_{\alpha^{\prime}}$} \\
\hline & & 22 & 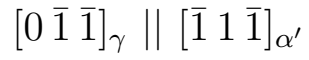 \\
\hline & & 23 & {$\left[\begin{array}{lll}1 & 0 & 1\end{array}\right]_{\gamma} \|\left[\begin{array}{lll}\overline{1} & \overline{1} & 1\end{array}\right]_{\alpha^{\prime}}$} \\
\hline & & 24 & {$\left[\begin{array}{lll}1 & 0 & 1\end{array}\right]_{\gamma} \|\left[\begin{array}{lll}\overline{1} & 1 & \overline{1}\end{array}\right]_{\alpha^{\prime}}$} \\
\hline
\end{tabular}




\section{References}

[1] B. J. Golden, D.-F. Li, Y. Guo, P. Tiernan, S. B. Leen, N. P. O’Dowd, Microscale deformation of a tempered martensite ferritic steel: Modelling and experimental study of grain and sub-grain interactions, Journal of the Mechanics and Physics of Solids 86 (2016) $42-52$.

[2] J. Dunlop, Y. M. Bréchet, L. Legras, Y. Estrin, Dislocation density-based modelling of plastic deformation of zircaloy-4, Materials Science and Engineering: A $443(1-2)(2007) 77-86$.

[3] Y. Li, V. Aubin, C. Rey, P. Bompard, Polycrystalline numerical simulation of variable amplitude loading effects on cyclic plasticity and microcrack initiation in austenitic steel 304l, International Journal of Fatigue 42 (2012) 71 - 81.

[4] A. L. Pécheur, F. Curtit, M. Clavel, J. Stephan, C. Rey, P. Bompard, Polycrystal modelling of fatigue: Pre-hardening and surface roughness effects on damage initiation for 304L stainless steel, International Journal of Fatigue 45 (2012) $48-60$.

[5] C. Frederick, P. Armstrong, A mathematical representation of the multiaxial bauschinger effect, Materials at High Temperatures 24 (1) (2007) $1-26$.

[6] J. Lemaitre, J.-L. Chaboche, Mechanics of solid materials, Cambrigde University Press, 1994.

[7] L. Tabourot, M. Fivel, E. Rauch, Generalised constitutive laws for f.c.c. single crystals, Materials Science and Engineering: A 234-236 (1997) 639 - 642.

[8] P. Franciosi, The concepts of latent hardening and strain hardening in metallic single crystals, Acta Metallurgica 33 (9) (1985) 1601 - 1612.

[9] M. Lee, H. Lim, B. Adams, J. Hirth, R. Wagoner, A dislocation density-based single crystal constitutive equation, International Journal of Plasticity 26 (7) (2010) $925-938$.

[10] B. Fournier, M. Sauzay, A. Pineau, Micromechanical model of the high temperature cyclic behavior of $9-12 \%$ Cr martensitic steels, International Journal of Plasticity 27 (11) (2011) 1803 - 1816.

[11] M. Sauzay, H. Brillet, I. Monnet, M. Mottot, F. Barcelo, B. Fournier, A. Pineau, Cyclically induced softening due to low-angle boundary annihilation in a martensitic steel, Materials Science and Engineering: A 400-401 (2005) 241 $-244$

[12] J. Hutchinson, Bounds and self-consistent estimates for creep of polycrystalline materials, Proceedings of the Royal Society of London A: Mathematical, Physical and Engineering Sciences 348 (1652) (1976) 101 - 127.

[13] R. Asaro, Crystal plasticity, Journal of Applied Mechanics 50 (4b) (1983) 921 $-934$. 
[14] R. Asaro, Micromechanics of crystals and polycrystals, in: J. W. H. a. T. Y. Wu (Ed.), Advances in Applied Mechanics, Vol. 23, Elsevier, 1983, pp. 1 - 115.

[15] D. Peirce, R. Asaro, A. Needleman, An analysis of nonuniform and localized deformation in ductile single crystals, Acta Metallurgica 30 (6) (1982) 1087 1119 .

[16] D. Peirce, R. Asaro, A. Needleman, Material rate dependence and localized deformation in crystalline solids, Acta Metallurgica 31 (12) (1983) 1951 - 1976.

[17] B. Xu, Y. Jiang, A cyclic plasticity model for single crystals, International Journal of Plasticity 20 (12) (2004) $2161-2178$.

[18] M. Berveiller, A. Zaoui, An extension of the self-consistent scheme to plasticallyflowing polycrystals, Journal of the Mechanics and Physics of Solids 26 (5-6) (1978) $325-344$.

[19] G. Cailletaud, P. Pilvin, Utilisation de modéles polycristallins pour le calcul par éléments finis, Revue Européenne des Eléments 3 (4) (1994) 515 - 541.

[20] F. Roters, P. Eisenlohr, L. Hantcherli, D. D. Tjahjanto, T. R. Bieler, D. Raabe, Overview of constitutive laws, kinematics, homogenization and multiscale methods in crystal plasticity finite-element modeling: Theory, experiments, applications, Acta Materialia 58 (4) (2010) 1152 - 1211.

[21] D. McDowell, Viscoplasticity of heterogeneous metallic materials, Materials Science and Engineering: R: Reports 62 (3) (2008) $67-123$.

[22] D. McDowell, A perspective on trends in multiscale plasticity, International Journal of Plasticity 26 (9) (2010) 1280 - 1309.

[23] J. Besson, G. Cailletaud, J.-L. Chaboche, S. Forest, M. Blétry, Non-linear mechanics of materials, Vol. 167 of Solid Mechanics and Its Applications, Springer, 2009.

[24] G. Cailletaud, S. Forest, D. Jeulin, F. Feyel, I. Galliet, V. Mounoury, S. Leroy, Some elements of microstructural mechanics, Computational Materials Science 27 (3) (2003) $351-374$.

[25] S. Forest, Milieux continus généralisés et matériaux hétérogénes, Presses de l'Ecole des Mines, 2006.

[26] O. Diard, S. Leclercq, G. Rousselier, G. Cailletaud, Evaluation of finite element based analysis of 3d multicrystalline aggregates plasticity: Application to crystal plasticity model identification and the study of stress and strain fields near grain boundaries, International Journal of Plasticity 21 (4) (2005) 691 - 722.

[27] E. Héripré, M. Dexet, J. Crépin, L. Gélébart, A. Roos, M. Bornert, D. Caldemaison, Coupling between experimental measurements and polycrystal finite element calculations for micromechanical study of metallic materials, International Journal of Plasticity 23 (9) (2007) 1512 - 1539. 
[28] Y. Guilhem, S. Basseville, F. Curtit, J.-M. Stéphan, G. Cailletaud, Numerical investigations of the free surface effect in three-dimensional polycrystalline aggregates, Computational Materials Science 70 (2013) 150 - 162.

[29] A. Guery, F. Hild, F. Latourte, S. Roux, Slip activities in polycrystals determined by coupling dic measurements with crystal plasticity calculations, International Journal of Plasticity 81 (2016) 249 - 266.

[30] C. Efstathiou, H. Sehitoglu, J. Lambros, Multiscale strain measurements of plastically deforming polycrystalline titanium: Role of deformation heterogeneities, International Journal of Plasticity 26 (1) (2010) 93 - 106.

[31] H. Lim, J. D. Carroll, C. C. Battaile, B. L. Boyce, C. R. Weinberger, Quantitative comparison between experimental measurements and cp-fem predictions of plastic deformation in a tantalum oligocrystal, International Journal of Mechanical Sciences 92 (2015) $98-108$.

[32] C. Gérard, G. Cailletaud, B. Bacroix, Modeling of latent hardening produced by complex loading paths in fcc alloys, International Journal of Plasticity 42 (2013) $194-212$.

[33] G. Cailletaud, A micromechanical approach to inelastic behaviour of metals, International Journal of Plasticity 8 (1) (1992) $55-73$.

[34] L. Méric, , P. Poubanne, G. Cailletaud, Single crystal modeling for structural calculations: Part 1-model presentation, Journal of Engineering Materials and Technology 113 (1) (1991) $162-170$.

[35] L. Méric, G. Cailletaud, Single crystal modeling for structural calculations: Part 2-finite element implementation, Journal of Engineering Materials and Technology 113 (1) (1991) $171-182$.

[36] A. Zouaghi, Modélisation multi-échelle du comportement non linéaire et hétérogéne en surface de l'acier AISI H11, Thése de doctorat, Université de Toulouse (2015).

[37] A. Zouaghi, V. Velay, A. Soveja, F. Rezai-Aria, A Numerical Investigation on the heterogeneous and anisotropic mechanical behaviour of AISI H11 steel using various stress-strain formulations: a multi-scale approach, in: 11th World Congress on Computational Mechanics, 2014, pp. 3415-3425.

[38] A. Alankar, D. P. Field, D. Raabe, Plastic anisotropy of electro-deposited pure $\alpha$-iron with sharp crystallographic $\left\langle\begin{array}{lll}1 & 1 & 1\end{array}\right\rangle / /$ texture in normal direction: Analysis by an explicitly dislocation-based crystal plasticity model, International Journal of Plasticity 52 (2014) 18 - 32.

[39] D. Cereceda, M. Diehl, F. Roters, D. Raabe, J. M. Perlado, J. Marian, Unraveling the temperature dependence of the yield strength in single-crystal tungsten using atomistically-informed crystal plasticity calculations, International Journal of Plasticity 78 (2016) $242-265$. 
[40] R. Quey, P. R. Dawson, F. Barbe, Large-scale 3D random polycrystals for the finite element method: Generation, meshing and remeshing, Computer Methods in Applied Mechanics and Engineering 200 (17-20) (2011) 1729 - 1745.

[41] C. Zhang, H. Li, P. Eisenlohr, W. Liu, C. Boehlert, M. Crimp, T. Bieler, Effect of realistic $3 \mathrm{~d}$ microstructure in crystal plasticity finite element analysis of polycrystalline ti-5al-2.5sn, International Journal of Plasticity 69 (2015) 21 35.

[42] D.-F. Li, B. J. Golden, N. P. O'Dowd, Multiscale modelling of mechanical response in a martensitic steel: A micromechanical and length-scale-dependent framework for precipitate hardening, Acta Materialia 80 (2014) 445 - 456.

[43] D. Raabe, M. Sachtleber, H. Weiland, G. Scheele, Z. Zhao, Grain-scale micromechanics of polycrystal surfaces during plastic straining, Acta Materialia 51 (6) (2003) $1539-1560$.

[44] Z. Zhao, M. Ramesh, D. Raabe, A. Cuitiño, R. Radovitzky, Investigation of three-dimensional aspects of grain-scale plastic surface deformation of an aluminum oligocrystal, International Journal of Plasticity 24 (12) (2008) 2278 2297.

[45] C. Tasan, J. Hoefnagels, M. Diehl, D. Yan, F. Roters, D. Raabe, Strain localization and damage in dual phase steels investigated by coupled in-situ deformation experiments and crystal plasticity simulations, International Journal of Plasticity 63 (2014) $198-210$.

[46] C. Tasan, M. Diehl, D. Yan, C. Zambaldi, P. Shanthraj, F. Roters, D. Raabe, Integrated experimental-simulation analysis of stress and strain partitioning in multiphase alloys, Acta Materialia 81 (2014) 386 - 400.

[47] S. Morito, Y. Adachi, T. Ohba, Morphology and crystallography of sub-blocks in ultra-low carbon lath martensite steel, Materials Transactions 50 (8) (2009) 1919 - 1923.

[48] H. Beladi, G. Rohrer, A. Rollett, V. Tari, P. Hodgson, The distribution of intervariant crystallographic planes in a lath martensite using five macroscopic parameters, Acta Materialia 63 (2014) 86 - 98.

[49] N. Mebarki, D. Delagnes, P. Lamesle, F. Delmas, C. Levaillant, Relationship between microstructure and mechanical properties of a $5 \% \mathrm{Cr}$ tempered martensitic tool steel, Materials Science and Engineering: A 387 - 389 (15) (2004) 171 -175 .

[50] V. Velay, G. Bernhart, D. Delagnes, L. Penazzi, A continuum damage model applied to high-temperalture fatigue lifetime prediction of a martensitic tool steel, Fatigue and Fracture of Eneneering Materials and Structures 28 (11) (2005) 1009-1023. 
[51] F. Krumphals, B. Reggiani, L. Donati, T. Wlanis, C. Sommitsch, Deformation behaviour of a ferritic hot-work tool steel with respect to the microstructure, Computational Materials Science 52 (1) (2012) 40 - 45.

[52] M. Poitrimolt, M. Cheikh, G. Bernhart, V. Velay, Characterisation of the transverse mechanical properties of carbon/carbon composites by spherical indentation, Carbon 66 (2014) $234-245$.

[53] R. Moulart, R. Rotinat, F. Pierron, Full-field evaluation of the onset of microplasticity in a steel specimen, Mechanics of Materials 41 (11) (2009) 1207 1222 .

[54] O. Barrau, C. Boher, R. Gras, F. Rézai-Aria, Analysis of the friction and wear behaviour of hot work tool steel for forging, Wear 255 (7-12) (2003) 1444 1454.

[55] C. Zambaldi, C. Zehnder, D. Raabe, Orientation dependent deformation by slip and twinning in magnesium during single crystal indentation, Acta Materialia 91 (2015) $267-288$.

[56] T. Bieler, P. Eisenlohr, F. Roters, D. Kumar, D. Mason, M. Crimp, D. Raabe, The role of heterogeneous deformation on damage nucleation at grain boundaries in single phase metals, International Journal of Plasticity 25 (9) (2009) $1655-1683$.

[57] C. Hamelin, B. Diak, A. Pilkey, Multiscale modelling of the induced plastic anisotropy in bcc metals, International Journal of Plasticity 27 (8) (2011) 1185 -1202 .

[58] A. Zeghadi, S. Forest, A.-F. Gourgues, O. Bouaziz, Ensemble averaging stressstrain fields in polycrystalline aggregates with a constrained surface microstructure - Part 2: crystal plasticity, Philosophical Magazine 87 (8-9) (2007) 1425 1446.

[59] T. Hoc, S. Forest, Polycrystal modelling of IF-ti steel under complex loading path, International Journal of Plasticity 17 (1) (2001) 65 - 85.

[60] A. Hlilou, I. B. Naceur, K. Saï, C. Gérard, S. Forest, G. Cailletaud, Generalization of the polycrystalline $\beta$-model: Finite element assessment and application to softening material behavior, Computational Materials Science 45 (4) (2009) $1104-1112$.

[61] V. Velay, G. Bernhart, L. Penazzi, Cyclic behavior modeling of a tempered martensitic hot work tool steel, International Journal of Plasticity 22 (3) (2006) $459-496$.

[62] Z. Zhang, D. Delagnes, G. Bernhart, Anisothermal cyclic plasticity modelling of martensitic steels, International Journal of Fatigue 24 (6) (2002) 635-648. 
[63] R. Foerch, J. Besson, G. Cailletaud, P. Pilvin, Polymorphic constitutive equations in finite element codes, Computer Methods in Applied Mechanics and Engineering 141 (3-4) (1997) 355 - 372.

[64] T. Erinosho, A. Cocks, F. Dunne, Texture, hardening and non-proportionality of strain in bcc polycrystal deformation, International Journal of Plasticity 50 (2013) $170-192$.

[65] S. Kuchnicki, A. Cuitiño, R. Radovitzky, Efficient and robust constitutive integrators for single-crystal plasticity modeling, International Journal of Plasticity 22 (10) (2006) $1988-2011$.

[66] S. Kuchnicki, R. Radovitzky, A. Cuitiño, An explicit formulation for multiscale modeling of bcc metals, International Journal of Plasticity 24 (12) (2008) 2173 $-2191$.

[67] H. Li, H. Yang, Z. Sun, A robust integration algorithm for implementing rate dependent crystal plasticity into explicit finite element method, International Journal of Plasticity 24 (2) (2008) $267-288$.

[68] J. Rossiter, A. Brahme, M. Simha, K. Inal, R. Mishra, A new crystal plasticity scheme for explicit time integration codes to simulate deformation in $3 \mathrm{~d}$ microstructures: Effects of strain path, strain rate and thermal softening on localized deformation in the aluminum alloy 5754 during simple shear, International Journal of Plasticity 26 (12) (2010) $1702-1725$.

[69] J. Segurado, R. A. Lebensohn, J. LLorca, C. Tomé, Multiscale modeling of plasticity based on embedding the viscoplastic self-consistent formulation in implicit finite elements, International Journal of Plasticity 28 (1) (2012) 124 140 .

[70] J. Simo, T. Hughes, Computational inelasticity, Springer-Verlag, New-York, 1998.

[71] A. M. Cuitiño, M. Ortiz, Computational modelling of single crystals, Modelling and Simulation in Materials Science and Engineering 1 (3) (1993) 225 - 263.

[72] F. Barbe, L. Decker, D. Jeulin, G. Cailletaud, Intergranular and intragranular behavior of polycrystalline aggregates. part 1: F.E. model, International Journal of Plasticity 17 (4) (2001) 513 - 536.

[73] F. Barbe, S. Forest, G. Cailletaud, Intergranular and intragranular behavior of polycrystalline aggregates.part 2: Results, International Journal of Plasticity 17 (4) (2001) $537-563$.

[74] F. Barbe, R. Quey, A numerical modelling of 3d polycrystal-to-polycrystal diffusive phase transformations involving crystal plasticity, International Journal of Plasticity 27 (6) (2011) $823-840$. 
[75] S. Morito, H. Tanaka, R. Konishi, T. Furuhara, T. Maki, The morphology and crystallography of lath martensite in Fe-C alloys, Acta Materialia 51 (6) (2003) $1789-1799$.

[76] S. Morito, X. Huang, T. Furuhara, T. Maki, N. Hansen, The morphology and crystallography of lath martensite in alloy steels, Acta Materialia 54 (19) (2006) $5323-5331$.

[77] C. Farhat, F.-X. Roux, A method of finite element tearing and interconnecting and its parallel solution algorithm, International Journal for Numerical Methods in Engineering 32 (6) (1991) 1205 - 1227.

[78] C. Boher, O. Barrau, R. Gras, F. Rezai-Aria, A wear model based on cumulative cyclic plastic straining, Wear 267 (5-8) (2009) 1087 - 1094. 